\title{
A Tractable Complex Network Model Based on the Stochastic Mean-Field Model of Distance
}

\author{
David J. Aldous \\ Department of Statistics \\ 367 Evans Hall \\ U.C. Berkeley CA 94720. \\ aldous@stat . berkeley . edu
}

November 10, 2018

\begin{abstract}
Much recent research activity has been devoted to empirical study and theoretical models of complex networks (random graphs) possessing three qualitative features: power-law degree distributions, local clustering, and slowly-growing diameter. We point out a new (in this context) platform for such models - the stochastic mean-field model of distances - and within this platform study a simple two-parameter proportional attachment (or copying) model. The model is mathematically natural, permits a wide variety of explicit calculations, has the desired three qualitative features, and fits the complete range of degree scaling exponents and clustering parameters; in these respects it compares favorably with existing models.
\end{abstract}

\section{Introduction}

The topic of complex networks, more precisely the design and theoretical analysis of stochastic models of large graphs which differ from the classical Erdős - Rényi model, has attracted intense recent attention, surveyed from a statistical physics viewpoint in [1, 10, 15] and from a rigorous mathematical viewpoint in [7].

Let us frame one aspect of this topic, by analogy. In freshman statistics we learn that bivariate data (e.g. heights and weights of $n$ individuals) can be summarized by 5 summary statistics: average height, standard deviation of height, average weight, standard deviation of weight, correlation coefficient. And there is a 5-parameter probability model, the bivariate Normal, which (in several precise senses) exactly corresponds to these particular summary statistics. In the context of real-world graphs (where we will always regard the number $n$ of vertices as large), one could analogously seek a crude statistical description by reporting a set of summary statistics. An evident choice is

- $\partial=$ average vertex-degree 
and recently popular extra choices include

- an exponent $\gamma$ characterizing power-law tail behavior of degree distribution

- a "clustering coefficient" $\kappa$ measuring relative density of triangles

- the average distance $\bar{\ell}$ between vertex-pairs.

These choices reflect and seek to quantify three qualitative features claimed to hold in many interesting graphs (from WWW links to human social networks): power-law degree distribution, local clustering of edges, and diameter growing as $O(\log n)$. So from the viewpoint of classical mathematical statistics, it would be natural to seek a several-parameter stochastic model of random graphs whose parameters could be readily identified with summary statistics of the kind above. In more detail, we propose three desiderata ${ }^{1}$ for a satisfactory model, beyond possessing the three qualitative features mentioned above:

- mathematical tractability: one can find reasonably explicit formulas for a variety of quantities of interest

- fitting flexibility: by varying model parameters one can vary summary statistics (like the 4 listed above) broadly through their possible ranges

- naturalness: the qualitative properties emerge from some simple underlying mathematical structure rather than being forced by fiat.

Unfortunately no satisfactory such models are known. The statistical physics literature surveyed in [1, 10] starts with a few elementary model-construction ideas (such as the proportional attachment and small worlds models mentioned in section 6) and then explores numerous variations. Our purpose in this paper is to introduce a new class of model we call metric copying, ${ }^{2}$ and to study a particular two-parameter model (mean-field simple copying, MFSC) within this class. The description and analysis of the MFSC model involve somewhat more sophisticated mathematical visualization than has been used in previous complex networks literature. So let us first address the first two desiderata by listing results for the model (section 21), and only later (section 3) describe the model. Section 4 derives most of the formulas in section 2 and section 5 exhibits further calculations. A briefer account of the model, aimed at mathematicians, appears in [4.

\subsection{Some notation}

$P(\cdot)$ denotes probability, $E(\cdot)$ denotes expectation, and var $(\cdot)$ denotes variance. We assume familiarity with elementary probability notions of random variables and their distributions. We write $\operatorname{Geo}(p), \operatorname{Bin}(m, p), \operatorname{Exp}(\mu), \operatorname{Poi}(\eta)$

\footnotetext{
${ }^{1}$ From an applied viewpoint, one could regard "fitting empirical data" as the single criterion; we are of course taking a theoretical viewpoint

${ }^{2}$ We use mathematical terminology: a metric is a distance function. Confusingly, some engineers use "metric" to mean "summary statistic"
} 
for the geometric, binomial, exponential and Poisson distributions in their usual parametrizations, reviewed below. We employ a "blackboard shorthand" of also writing $\operatorname{Geo}(p)$ etc for a random variable with that distribution. Thus the elementary reproductive property of the binomial distribution could be written as

$$
\operatorname{Bin}\left(m_{1}+m_{2}, p\right) \stackrel{d}{=} \operatorname{Bin}\left(m_{1}, p\right)+\operatorname{Bin}\left(m_{2}, p\right)
$$

where the random variables on the right are independent, and where $\stackrel{d}{=}$ means equality in distribution. The point of this notation is that, analogous to "composition of functions" in which we interpret $\exp \left((x-1)^{2}\right)$ as the composition of the two functions $\exp (x)$ and $(x-1)^{2}$, we can "compose" (statisticians say "mix") distributions. For instance (cf. (8) below), given a random variable $\Lambda$ with values in $(0,1)$ we can write $\operatorname{Geo}(\Lambda)$ for a random variable whose conditional distribution given $\Lambda=p$ is the $\operatorname{Geo}(p)$ distribution.

Review of elementary distributions.

$$
\begin{aligned}
P(\operatorname{Geo}(p)=i) & =(1-p)^{i-1} p, i=1,2, \ldots \\
E \operatorname{Geo}(p) & =p^{-1} \\
P(\operatorname{Bin}(m, p)=i) & =\left(\begin{array}{c}
m \\
i
\end{array}\right) p^{i}(1-p)^{m-i}, i=0,1, \ldots, m \\
E \operatorname{Bin}(m, p) & =m p \\
\operatorname{var} \operatorname{Bin}(m, p) & =m p(1-p) \\
P(\operatorname{Poi}(\eta)=i) & =e^{-\eta} \eta^{i} / i !, i=0,1,2, \ldots \\
E \operatorname{Poi}(\eta) & =\eta .
\end{aligned}
$$

The $\operatorname{Exp}(\mu)$ distribution has probability density function and expectation

$$
\begin{aligned}
f(x) & =\mu e^{-\mu x}, 0<x<\infty \\
\operatorname{EExp}(\mu) & =\mu^{-1} .
\end{aligned}
$$

A Poisson process of rate 1 , say $\left(0<\xi_{1}<\xi_{2}<\xi_{3}<\ldots\right)$, is defined by the property

$$
\xi_{1}, \xi_{2}-\xi_{1}, \xi_{3}-\xi_{2}, \ldots \text { are independent with } \operatorname{Exp}(1) \text { distribution }
$$

and has the property

$$
P\left(\text { some } \xi_{i} \in[x, x+d x]\right)=1 \cdot d x, 0<x<\infty .
$$

\subsection{Organization of paper}

Because the precise definition and a priori motivation of the model are lengthy to explain, we start by emphasising the a posteriori motivation, the fact that the model permits many explicit calculations. In first reading the formulas in section 2 focus on the left sides of equations, indicating what quantities can be calculated. The formulas on the right sides will be derived in section 4 


\section{Formulas}

\section{$2.1 \quad$ Key methodology}

Like other models involving vertices arriving and creating edges to existing vertices, the MFSC model defines a directed acyclic (no directed cycles) random graph $\mathcal{G}_{n}$ on $n$ vertices. A key feature of the model is that there exists a well-defined limit infinite rooted graph $\mathcal{G}_{\infty}^{*}$ which represents the $n \rightarrow \infty$ limit of $\mathcal{G}_{n}$ rooted at a uniform random (we say "typical") vertex. So for "local" statistics of $\mathcal{G}_{n}$, one can give "exact formulas in the $n \rightarrow \infty$ limit" by doing calculations within the limit structure $\mathcal{G}_{\infty}^{*}$, and this methodology is how we will derive (section 4) and interpret the formulas in sections 2.2 - 2.6] below.

Note that "rooting" is introduced merely as a convenient technical way to deal with infinite graphs. By analogy, one could study two-dimensional space without introducing the origin point (cf. Euclidean geometry) but for many purposes an origin and induced coordinate system are helpful.

The MFSC model has two parameters: $\alpha, \lambda$. In explicit formulas, we distinguish between a low clustering region defined by parameter ranges

$$
0<\alpha<1, \quad 0<\lambda \leq 1 / \alpha \quad[\text { low }]
$$

and the complementary high clustering region defined by $\alpha \lambda>1$; in the latter case it is convenient to reparametrize by using $\eta:=\lambda^{-1} \log (\alpha \lambda)$ in place of $\alpha$, and the parameter ranges are

$$
0<\eta<1, \quad \eta+1 / \lambda<1 \quad \text { [high] }
$$

This distinction is purely notational; there is no intrinsic "non-analyticity" in the model's properties.

\subsection{The two parameters control mean degree and cluster- ing}

(a). First consider $D_{\text {in }}$ and $D_{\text {out }}$, the random in-degree and out-degree of a typical vertex. Then

$$
E D_{\text {in }}=E D_{\text {out }}(=\partial, \text { say })=\left\{\begin{array}{cc}
\frac{\alpha}{1-\alpha} & {[\text { low }]} \\
\frac{\eta+1 / \lambda}{1-\eta-1 / \lambda} & {[\text { high }]}
\end{array}\right.
$$

(b). Second, define a normalized clustering coefficient $\kappa_{\text {cluster }}$ in words as

The proportion of directed 2-paths $v_{1} \rightarrow v_{2} \rightarrow v_{3}$ for which $v_{1} \rightarrow v_{3}$ is also an edge.

(see (44) for a more precise definition and derivation of (7)). Then

$$
\kappa_{\text {cluster }}=\left\{\begin{array}{cc}
\frac{\alpha(1-\alpha) \lambda}{2-\alpha^{2} \lambda} & {[\text { low }]} \\
\frac{\left(\eta+\frac{1}{2 \lambda}\right)\left(1-\eta-\frac{1}{\lambda}\right)}{\left(\eta+\frac{1}{\lambda}\right)\left(1-\eta-\frac{1}{2 \lambda}\right)} & {[\text { high }]}
\end{array}\right.
$$


By solving (617) we find (section 4.6) that every pair of values of $\partial, \kappa_{\text {cluster }}$ in the complete range

$$
0<\partial<\infty, \quad 0<\kappa_{\text {cluster }}<1
$$

occurs for a unique parameter pair $(\alpha, \lambda)$ or $(\eta, \lambda)$. Moreover the two regions can be specified as

$$
\begin{array}{lll}
0<\partial<\infty, & 0<\kappa_{\text {cluster }} \leq \frac{1}{\partial+2} & {[\text { low }]} \\
0<\partial<\infty, & \frac{1}{\partial+2}<\kappa_{\text {cluster }}<1 & {[\text { high }]}
\end{array}
$$

explaining our low and high clustering terminology. So the two model parameters $\alpha, \lambda$ have fairly direct interpretations in terms of mean degree and clustering; of course we could re-parametrize the model in terms of $\partial$ and $\kappa_{\text {cluster }}$, but the internal mathematical structure is more conveniently expressed using the given parameters.

\subsection{Distributions of in- and out-degrees}

(a). The distribution of $D_{\text {in }}$ is specified as

$$
1+D_{\text {in }} \stackrel{d}{=} \operatorname{Geo}\left(e^{-\beta T}\right) \text { where } T \stackrel{d}{=} \operatorname{Exp}(1)
$$

and where

$$
\beta=\left\{\begin{array}{cc}
\alpha & {[\text { low }]} \\
\eta+1 / \lambda & {[\text { high }]}
\end{array}\right.
$$

This works out explicitly as

$$
\begin{array}{ll}
P\left(D_{\text {in }} \geq d\right)=\frac{\Gamma(d+1) \Gamma(1 / \beta)}{\beta \Gamma\left(d+1+\frac{1}{\beta}\right)}, & d \geq 0 \\
P\left(D_{\text {in }}=d\right)=\frac{\Gamma(d+1) \Gamma(1 / \beta)}{\beta^{2} \Gamma\left(d+2+\frac{1}{\beta}\right)}, & d \geq 0
\end{array}
$$

with asymptotics

$$
P\left(D_{\text {in }}=d\right) \sim \beta^{-2} \Gamma(1 / \beta) d^{-1-\frac{1}{\beta}} .
$$

Formula (11) appears as a special case of recent results in two-parameter proportional attachment models [9, 11, 13, but in fact is a famous 80-year old calculation - see section 4.2

(b). The distribution of $D_{\text {out }}$ is determined by the distributional equation

$$
D \stackrel{d}{=}\left\{\begin{array}{cc}
\sum_{i=1}^{\infty} \operatorname{Bin}\left(1+D_{i}, \alpha \lambda e^{-\lambda \xi_{i}}\right) & {[\mathrm{low}]} \\
\sum_{i=1}^{\operatorname{Poi}(\eta)}\left(1+D_{i}^{\prime}\right)+\sum_{i=1}^{\infty} \operatorname{Bin}\left(1+D_{i}, e^{-\lambda \xi_{i}}\right) & {[\text { high }]}
\end{array}\right.
$$

where $D, D_{i}, D_{i}^{\prime}, i \geq 1$ are i.i.d. random variables distributed as $D_{\text {out }}$ and where $0<\xi_{1}<\xi_{2}<\ldots$ are the points of a rate-1 Poisson point process on $(0, \infty)$. 
We do not know how to extract a useful explicit formula from (12) but we can compute moments. For instance

$$
\text { var } D_{\text {out }}= \begin{cases}\frac{\alpha\left(1-\alpha+\alpha^{2} \lambda / 2\right)}{(1-\alpha)^{2}\left(1-\frac{1}{2} \alpha^{2} \lambda\right)} & {[\text { low }]} \\ \frac{\left(\eta+\frac{1}{2 \lambda}\right)\left(2-\eta-\frac{1}{\lambda}\right)}{\left(1-\eta-\frac{1}{2 \lambda}\right)\left(1-\eta-\frac{1}{\lambda}\right)^{2}} & {[\text { high }]}\end{cases}
$$

In the case $\lambda=1 / \alpha$ and in the limits $\lambda \rightarrow 0, \lambda \rightarrow \infty$ we get explicit formulas for the distribution of $D_{\text {out }}$ - see section 4.3 - which show in particular that the tail of $D_{\text {out }}$ has geometric rather than power-law decay.

(c).

$$
D_{\text {in }} \text { and } D_{\text {out }} \text { are independent. }
$$

Because both $D_{\text {out }}$ and $D_{\text {in }}$ can take the value 0 , we see that $P\left(D_{\text {in }}+D_{\text {out }}=\right.$ $0)>0$, implying that $\mathcal{G}_{n}$ will typically not be connected (see section 2.7 for further comments).

\subsection{Densities of induced subgraphs}

One of the major advantages of the model is that, for a fixed "small" graph $G$, one can (in principle, and often in practice) calculate explicitly an "asymptotic density" $\operatorname{dens}_{\infty}(G)$ interpreted as the limit

$$
\lim _{n \rightarrow \infty} \frac{\text { number of copies of } G \text { in } \mathcal{G}_{n}}{n}=\operatorname{dens}_{\infty}(G) .
$$

Precise definitions are fussy, and are deferred to section 4.4 which also records the explicit formulas we have found. Here let us point out the formula for triangles $K_{3}$ :

$$
\operatorname{dens}_{\infty}\left(K_{3}\right)= \begin{cases}\frac{\alpha^{3} \lambda}{(1-\alpha)\left(2-\alpha^{2} \lambda\right)} & {[\text { low }]} \\ \frac{\left(\eta+\frac{1}{\lambda}\right)\left(\eta+\frac{1}{2 \lambda}\right)}{\left(1-\eta-\frac{1}{\lambda}\right)\left(1-\eta-\frac{1}{2 \lambda}\right)} & {[\text { high }] .}\end{cases}
$$

The formula above is the key ingredient in the formula for $\kappa_{\text {cluster }}$. Recall the verbal description of $\kappa_{\text {cluster: }}$ :

The proportion of directed 2-paths $v_{1} \rightarrow v_{2} \rightarrow v_{3}$ for which $v_{1} \rightarrow v_{3}$ is also an edge.

It is intuitively clear (and formalized at (40) that the asymptotic density for occurrence of directed 2-paths $v_{1} \rightarrow v_{2} \rightarrow v_{3}$, if one does not look whether or not a third edge $v_{1} \rightarrow v_{2}$ is present, equals $\partial^{2}$ (because of independence of in-degree and out-degree at $v_{2}$ ). So the verbal definition translates to

$$
\kappa_{\text {cluster }}=\frac{\operatorname{dens}_{\infty}\left(K_{3}\right)}{\partial^{2}}
$$

and then (15) immediately gives formula (7) for $\kappa_{\text {cluster. }}$ 


\subsection{Triangle density as a function of degree}

The parameter $\kappa_{\text {cluster }}$ gives an overall measure of triangle density. A more detailed description is provided by statistics $C(k), k \geq 2$ defined by

$$
C(k)=\frac{E \text { (number of triangles containing a random degree- } k \text { vertex) }}{\left(\begin{array}{c}
k \\
2
\end{array}\right)} .
$$

In principle the methods of this paper could be used to obtain an exact formula for $C(k)$, but we shall be content with outlining (section [5.5) the tail property

$$
C(k) \sim \frac{2 \beta_{2}}{\beta-\beta_{2}} \times \frac{1}{k} \text { as } k \rightarrow \infty .
$$

See section $[6$ for further comments.

\subsection{Edge-lengths}

Our model has a "metric structure", in that there is a distance $d_{\text {metric }}(v, w)$ between any two vertices which does not involve the realization of edges in the random graph. So each edge $(v, w)$ of the graph has a real-valued length $d_{\text {metric }}(v, w)$, and so a typical edge has a random length $L$. The probability density function for $L$ is given by the formula

$$
f(x)=\frac{1-\alpha}{\alpha} \sum_{i=0}^{\infty} \frac{(i+1) \Gamma(\alpha+3)(-\lambda x)^{i}}{\Gamma(i+\alpha+3)}, 0<x<\infty \quad[\text { low }] .
$$

Mathematica gives an equivalent expression as a sum of incomplete hypergeometric functions. One can readily observe that $f(x)=\exp (-(\lambda \pm o(1)) x)$ as

$x \rightarrow \infty$. In the underlying metric space, the number of vertices within distance $x$ of a typical vertex grows as $e^{x}$. So the tail behavior of $f(x)$ suggests

the chance that a vertex has an edge to its $k$ 'th nearest neighbor should scale as $k^{-\lambda-1}$

though we have not attempted detailed calculations to verify this suggestion. Note this property appears without being explicitly built into the model.

\subsection{Other local statistics}

There are further questions, concerning exact behavior in the $n \rightarrow \infty$ limit, which are in principle solvable in terms of the limit network $\mathcal{G}_{\infty}^{*}$, but where we have been unable to obtain usefully explicit answers. A major question concerns the percolation probability

$$
p_{\text {perc }}(\alpha, \lambda)=P\left(\text { typical vertex is in infinite connected component of } \mathcal{G}_{\infty}^{*}\right)
$$


By analogy with classical facts about the Erdős - Rényi model, we expect that above the percolation threshold, that is when $p_{\text {perc }}(\alpha, \lambda)>0$, the random graph $\mathcal{G}_{n}$ will have a giant component whose size $C_{n}(\alpha, \lambda)$ satisfies

$$
n^{-1} E C_{n}(\alpha, \lambda) \rightarrow p_{\text {perc }}(\alpha, \lambda) .
$$

Unfortunately we do not see how to write $p_{\text {perc }}(\alpha, \lambda)$ as a solution of any simple equation. By studying an easier-to-analyze directed percolation problem, it is not hard to show (section 5.3)

$$
\text { if } 2 \beta-\beta_{2}>1 \text { then } p_{\text {perc }}(\alpha, \lambda)>0 .
$$

\subsection{Average distance}

In any graph, write $d_{\text {graph }}(v, w)$ for the minimal number of edges in any path from $v$ to $w$. The diameter $\Delta$ and the average vertex-vertex distance $\Lambda$ are defined by

$$
\Delta=\max _{v, w} d_{\text {graph }}(v, w), \Lambda=\operatorname{ave}_{v, w} d_{\text {graph }}(v, w) .
$$

In the context of a simple proportional attachment model it is known that

$$
E \Delta_{n}, E \Lambda_{n}=\frac{(1+o(1)) \log n}{\log \log n} \text { as } n \rightarrow \infty .
$$

It is natural to conjecture, but hard to prove, the same result for our model (above the percolation threshold and restricted to the giant component). On the other hand it seems likely that standard techniques of abstract mathematical probability would be enough to show the weaker bound $E \Lambda_{n}=O(\log n)$ as $n \rightarrow$ $\infty$. Such questions cannot in principle be answered completely using $\mathcal{G}_{\infty}^{*}$.

\subsection{Summary of advantages and disadvantages of the model}

The previous sections convey some advantages of the model:

- it has the three qualitative features desired in a complex network model (power-law degree distribution, clustering, small diameter);

- it fits the complete possible range of mean degree (or scaling exponent) and clustering parameters;

- it permits a broad range of explicit calculations.

So to be fair let us list some disadvantages from a modeling viewpoint.

- $\mathcal{G}_{n}$ is not connected (for large $n$ ); cf. section 2.7

- there is no power law for distribution of out-degree;

- in-degree and out-degree are independent; 
- the scaling exponent for in-degree is determined by the mean degree; one might prefer a model where these could be specified separately;

- in the $n \rightarrow \infty$ limit not every finite graph is possible as an induced subgraph (section 4.5).

\section{The model}

\subsection{Metric copying models}

Let us briefly outline a general modeling framework, metric copying models. Each vertex $v$ is a point in a metric space; that is, there is some real-valued distance $d(v, w)$ between any two vertices $v, w$. Given some rule for the positions of successive vertices $1,2, \ldots$, and given a function $p:[0, \infty) \rightarrow[0,1]$, we can construct random directed graphs $\mathcal{G}_{n}$ inductively on $n$ as follows. When vertex $n$ arrives, then

(i) for each directed edge $(i, j)$ of $\mathcal{G}_{n-1}$, a "copied" edge $(n, j)$ is created with probability $p(d(n, i))$;

(ii) for each vertex $i(1 \leq i<n)$, a new edge $(n, i)$ is created with probability $p(d(n, i))$;

(iii) the events above are independent, except that repeat edges are censored.

Imagine $p(\cdot)$ to be rapidly decreasing. A moment's thought shows how this model resembles proportional attachment models. An existing vertex $v$ with indegree $d$ has $d+1$ opportunities to acquire an in-edge, due to the next arriving vertex being close to $v$ or close to one of the $d$ vertices with edges to $v$.

In principle one could study such models based on random points in $d$ dimensional space, but within such settings it is notoriously hard to do explicit calculations (see e.g. [16] for different models of random graphs based on $d$ dimensional random points), and the choice of $d$ is arbitrary. We will avoid both problems by using a well known (in other contexts) model which is loosely interpretable as "random points in infinite-dimensional space". Note that in $d$-dimensional space, the number of points within distance $r$ of a typical point grows as $r^{d}$; what will make our model "infinite-dimensional" is that this number grows as $e^{r}$.

For later use recall that a pre-metric $\bar{d}(i, j)$ is symmetric and strictly positive for $j \neq i$. A pre-metric can be used to specify a metric $d(v, w)$ as the minimum, over paths $v=i_{0}, i_{1}, \ldots, i_{k}=w$, of $\bar{d}\left(i_{0}, i_{1}\right)+\bar{d}\left(i_{1}, i_{2}\right)+\ldots+\bar{d}\left(i_{k-1}, i_{k}\right)$.

\section{$3.2 \quad$ A $d$-dimensional analogy}

As a final preliminary, the following analogy may be helpful. In $d$-dimensional space $R^{d}$, take a cube $\left[-n^{1 / d} / 2, n^{1 / d} / 2\right]^{d}$ of volume $n$, and put $n$ uniform random points in that cube. This structure has a $n \rightarrow \infty$ limit, the Poisson point process in $R^{d}$ with mean intensity 1 point per unit volume. Moreover the limit process, which is a spatial point process on all of $R^{d}$, can be represented as the 
distribution, at any fixed time, of a time-evolving process of points on all of $R^{d}$, where the evolution rules are

(i) points move away from the origin as deterministic motion with exponential rate $1 / d$; a point at position $x$ at time $t$ will be at position $x e^{\left(t^{\prime}-t\right) / d}$ at times $t^{\prime}>t$.

(ii) New points arrive throughout $R^{d}$ as a rate- 1 space-time Poisson process; that is, the chance of a point arriving in a cube of volume $d x$ during a time interval $d t$ equals $1 \cdot d x d t$.

Thus if one takes a volume- 1 region of space at time $t_{0}$, this space expands to become volume $e^{t}$ at time $t_{0}+t$, and the arrival rate per unit time within this expanding volume is $e^{t}$ at time $t_{0}+t$.

In the limit process, one may regard the "present time" as time 0 , and regard the process as having evolved ${ }^{3}$ over time $-\infty<t \leq 0$. Particles at the present time have ages which are independent $\operatorname{Exp}(1)$ random variables independent of present positions; from the present configuration of positions and ages one can deterministically reconstruct the past evolution of the process.

\subsection{The stochastic mean-field model of distance}

Our model of an underlying metric space is specified by three rules.

(i) Point $n$ arrives at time $t_{n}=\log n$.

(ii) At the arrival time $t_{n}$, define the pre-distances $\left(\bar{D}\left(n, j ; t_{n}\right), 1 \leq j \leq n-1\right)$ from $n$ to the earlier-arriving points to be independent random variables with exponential, mean $n$, distribution.

(iii) Distances grow exponentially with time; $\bar{D}(n, j ; t)=e^{t-t_{n}} \bar{D}\left(n, j ; t_{n}\right)$ for $t>t_{n}$.

So at time $t$ there are $n=\left\lfloor e^{t}\right\rfloor$ points, and the $\left(\begin{array}{l}n \\ 2\end{array}\right)$ pre-distances $\bar{D}(i, j ; t), 1 \leq$ $i<j \leq n$ ) are independent random variables with exponential, mean $e^{t}$, distribution. These particular pre-distances are an instance of a pre-metric, and this pre-metric specifies a metric $D(i, j ; t), 1 \leq i<j \leq n)$. Write $\left(\mathcal{D}_{t}, 0 \leq t<\infty\right)$ for this process of arriving points and distances.

Here is a key feature of this construction. At time $t$ pick a uniform random point $V_{t}$ as a "root". Then there is a $t \rightarrow \infty$ limit (in distribution) structure, which is a metric space on a countable infinite number of points, one being distinguished as the root. The limit structure, called the PWIT, is described below. The meaning of "limit" is that, for arbitrary fixed $r<\infty$, the configuration of points in $\mathcal{D}_{t}$ within distance $r$ of $V_{t}$ converges in distribution to the configuration of points of the PWIT within distance $r$ of the root (this is local weak convergence of random networks [6]).

\footnotetext{
${ }^{3}$ This model is reminiscent of the steady-state theory of the Universe advocated by Fred Hoyle in the 1950s
} 


\subsection{The PWIT}

The PWIT is defined by a construction, illustrated in Figure $1^{4}$. Start with a single root vertex $\emptyset$. This root vertex is then given an infinite number of near neighbors, and the edges from the root to the near neighbors are assigned lengths according to a realization of a Poisson process $\left(\xi_{i}^{\emptyset}: 1 \leq i<\infty\right)$ of rate 1 on $(0, \infty)$. Now, recursively, each vertex $v$ arising as a near neighbor of a previous vertex is given an infinite number of near neighbors, and the edges to these near neighbors of $v$ are again assigned lengths according to an independent realization of a Poisson process $\left(\xi_{i}^{v}: 1 \leq i<\infty\right)$ of rate 1 . This procedure is then continued ad infinitum. The resulting rooted infinite tree is a well defined random object, called the Poisson weighted infinite tree (PWIT).

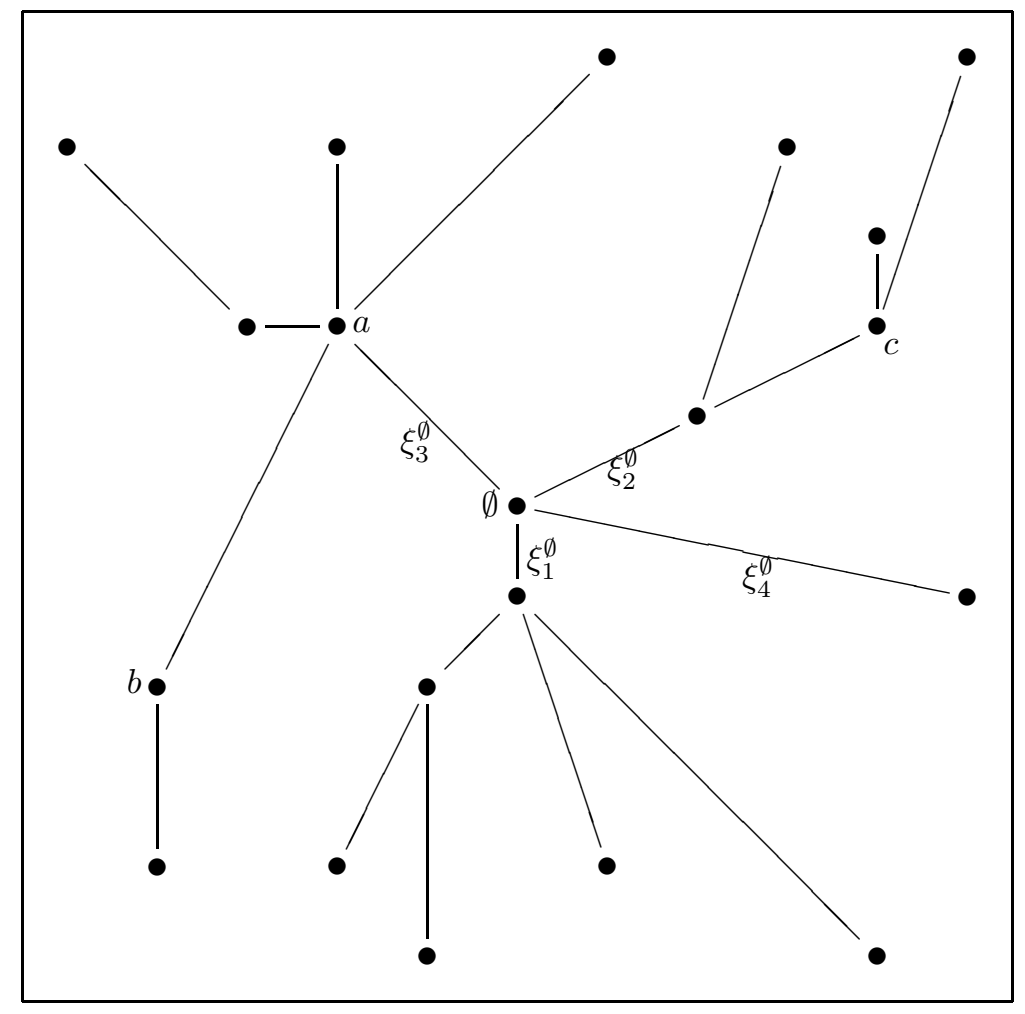

Figure 1. The PWIT. Illustration of the vertices of the PWIT within a window of radius 3 centered on the root $\emptyset$. Lines indicate the near neighbor relationship, but are drawn only when both end-vertices are within the window. Thus the four near neighbors of $\emptyset$ shown are at distances $0<\xi_{1}^{\emptyset}<\xi_{2}^{\emptyset}<\xi_{3}^{\emptyset}<\xi_{4}^{\emptyset}<3$ from $\emptyset$, while there are an infinite number of near neighbors of $\emptyset$ at distances greater than 3 . Orientation of lines in pictures is arbitrary. Labels $a, b, c$ are included for later comparisons.

\footnotetext{
${ }^{4}$ Our figures are illustrations of the definitions, rather than honest Monte Carlo simulations
} 
The distance $D(v, w)$ between two vertices of the PWIT is just the sum of edgelengths along the path from $v$ to $w$. Though we have drawn a tree in Figure 1, the lines merely indicate the near neighbor relationships; it is better to think of the edges as absent while retaining the distances $D(v, w)$. In this way we may regard the vertices of the PWIT as an infinite-dimensional analog of the $d$-dimensional Poisson point process in section 3.2 Formula (29) later provides one formalization of "infinite-dimensional".

The survey [6] gives a careful explanation of how the PWIT arises as a limit of finite models such as $\mathcal{D}_{t}$, and gives some applications to combinatorial optimization $^{5}$. The key point is that, for an arriving vertex $V_{t}$ in $\mathcal{D}_{t}$, the existing vertices at smallest $\bar{D}$-distances correspond in the limit to the near neighbors in the PWIT. (Recall the section 3.3 construction; we are repeating the "key feature" from the last paragraph of that section.)

In the present setting, each point $v$ of $\mathcal{D}_{t}$ has an "age" at time $t$, and in the limit PWIT these ages are (exactly as in section 3.2) independent $\operatorname{Exp}(1)$ random variables, $A_{v}$ say. Thus if we write $\mathcal{D}_{0}^{*}$ for the PWIT and $A_{\text {root }}$ for the age of the root, then (given the other ages $A_{v}$ also) we can reconstruct the time-evolution of a backwards space-time PWIT process $\left(\mathcal{D}_{s}^{*},-A_{\text {root }} \leq s \leq 0\right)$. Precisely, as $s$ runs backwards

(a) the edge-lengths $\xi$ decrease exponentially; at time $s<0$ the length is $\xi e^{s}$;

(b) a vertex $v$ and its incident edges are deleted at $s=-A_{v}$.

Then $\mathcal{D}_{s}^{*}$ is defined as the connected component containing the root at time $s$. This limit process relates to the finite process as follows. Let $\tilde{A}_{t}$ be the age (at time $t$ ) of the randomly-chosen vertex $V_{t}$ at time $t$. Then

$$
\left(\mathcal{D}_{t+s}^{*(t)},-\tilde{A}_{t} \leq s \leq 0\right) \stackrel{d}{\rightarrow}\left(\mathcal{D}_{s}^{*},-\tilde{A}_{\text {root }} \leq s \leq 0\right) \text { as } t \rightarrow \infty
$$

where $\mathcal{D}_{t+s}^{*(t)}$ is the configuration $\mathcal{D}_{t+s}$ rooted at $V_{t}$.

There is also a forwards space-time PWIT process $\left(\mathcal{D}_{s}^{*}, 0 \leq s<\infty\right)$ specified as follows. Start with the PWIT $\mathcal{D}_{0}^{*}$. At time $s$ increases, all inter-vertex distances increase at exponential rate 1 . For each vertex $v$ present at time $s$, and each $0<r<\infty$, there is (as explained below) chance $1 \cdot d r d s$ that during $[s, s+d s]$ a new vertex $v^{\prime}$ will appear at distance $\in[r, r+d r]$ from $v$ as a near neighbor of $v$. Along with this vertex (which has current age 0) is an independent copy of the PWIT rooted at $v^{\prime}$, whose other vertex-ages are independent $\operatorname{Exp}(1)$. The relation between the finite- $t$ and the limit process is analogous to (20):

$$
\left(\mathcal{D}_{t+s}^{*(t)}, 0 \leq s<\infty\right) \stackrel{d}{\rightarrow}\left(\mathcal{D}_{s}^{*}, 0 \leq s<\infty\right) \text { as } t \rightarrow \infty .
$$

Here is the calculation leading to the coefficient " 1 " in

there is chance $1 \cdot d r d s$ that during $[s, s+d s]$ a new vertex $v^{\prime}$

will appear at distance $\in[r, r+d r]$ from $v$ as a near neighbor of $v$.

\footnotetext{
${ }^{5}$ See also 3 for novel scaling exponents arising in the study of the mean-field traveling salesman problem.
} 
In the process $\left(\mathcal{D}_{t}\right)$, during time $[t+s, t+s+d s]$ about $e^{t+s} d s$ vertices arrive; for each existing vertex, the chance an arriving vertex is within $\bar{D}$-distance $[r, r+d r]$ equals $e^{-(t+s)} \exp \left(-r e^{-(t+s)}\right) d r$. So the chance in (22) equals

$$
e^{t+s} d s \times e^{-(t+s)} \exp \left(-r e^{-(t+s)}\right) d r \approx 1 \times d r d s .
$$

Recursive self-similarity. Implicit in the model is the fact that the "geometry" of the space seen by a newly-arriving particle $v^{*}$ is statistically the same as the geometry seen by a typical existing particle. This is the familiar PASTA (Poisson arrivals see time averages) property in queuing theory. In particular, at the arrival time of $v^{*}$ the geometric components containing the different near neighbor vertices $v_{1}, v_{2}, \ldots$ are independent copies of the PWIT. This recursive self-similarity property of the PWIT process is fundamental to its analytic tractability.

Figure 2 and its legend may be helpful.
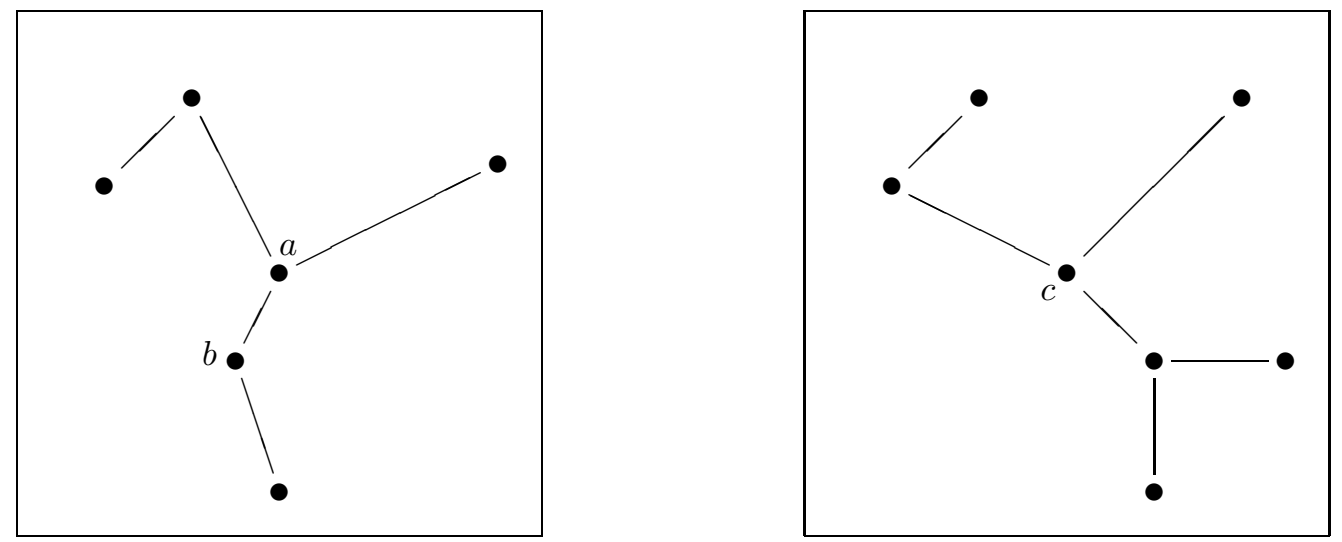

Figure 2. The space-time PWIT process. Regarding Figure 1 as showing the PWIT at a time $t_{+}$, Figure 2 shows the space-time PWIT at an earlier time $t_{-}$ at which only three vertices $a, b, c$ of the vertices in the Figure 1 window have arrived. Figure 2 shows smaller windows centered on $a$ and on $c$. The other vertices in Figure 2 , and the near neighbor relation shown by lines, are still present at time $t_{+}$, but are not visible in Figure 1 because the expansion of distances has placed them outside the Figure 1 window.

\subsection{The MFSC model}

The process $\left(\mathcal{D}_{t_{n}}, n=1,2,3, \ldots\right)$ of arrivals and inter-point distances described in section 3.3 defines an "underlying geometry"; we now define the random graph process $\left(\mathcal{G}_{n}, n=1,2,3, \ldots\right)$ which is the subject of this paper. Fix two parameters $0<\alpha<\infty$ and $0<\lambda<\infty$. Write

$$
p(x)=\min \left(1, \alpha \lambda e^{-\lambda x}\right), \quad 0 \leq x<\infty .
$$


We now implement a version of the "metric copying" idea from section $3.1 \mathcal{G}_{1}$ consists of vertex 1 and no edges. When vertex $n$ arrives at time $t_{n}=\log n$, then

(i) for each directed edge $(i, j)$ of $\mathcal{G}_{n-1}$, a "copied" edge $(n, j)$ is created with probability $p\left(\bar{D}\left(n, i ; t_{n}\right)\right)$;

(ii) for each vertex $i(1 \leq i<n)$, a new edge $(n, i)$ is created with probability $p\left(\bar{D}\left(n, i ; t_{n}\right)\right)$;

(iii) the events above are independent, except that repeat edges are censored.

Note that we use $\bar{D}$ instead of $D$ in determining attachment probabilities (because $\bar{D}$-near vertices at finite time correspond to the near neighbors in the limit PWIT). We call $\left(\mathcal{G}_{n}, n=1,2,3, \ldots\right)$ the mean-field simple copying (MFSC) model.
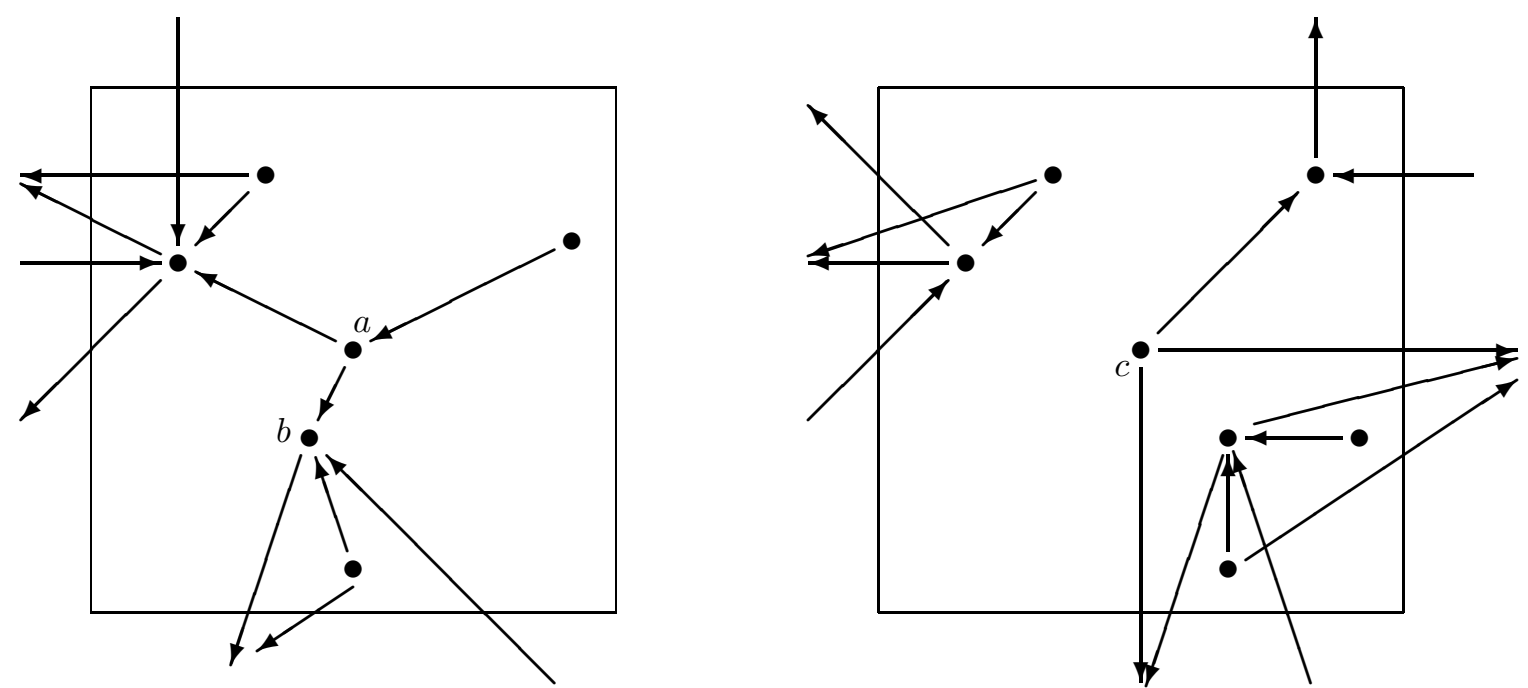

Figure 3. The graph process $\mathcal{G}_{\infty}^{*}\left(t_{-}\right)$on the realization of the space-time PWIT at time $t_{-}$in Figure 2. For the graph process we show all edges with either endvertex within the window. The following figures show the evolution of $\mathcal{G}_{\infty}^{*}(t)$ over $t_{-}<t \leq t_{+}$. 


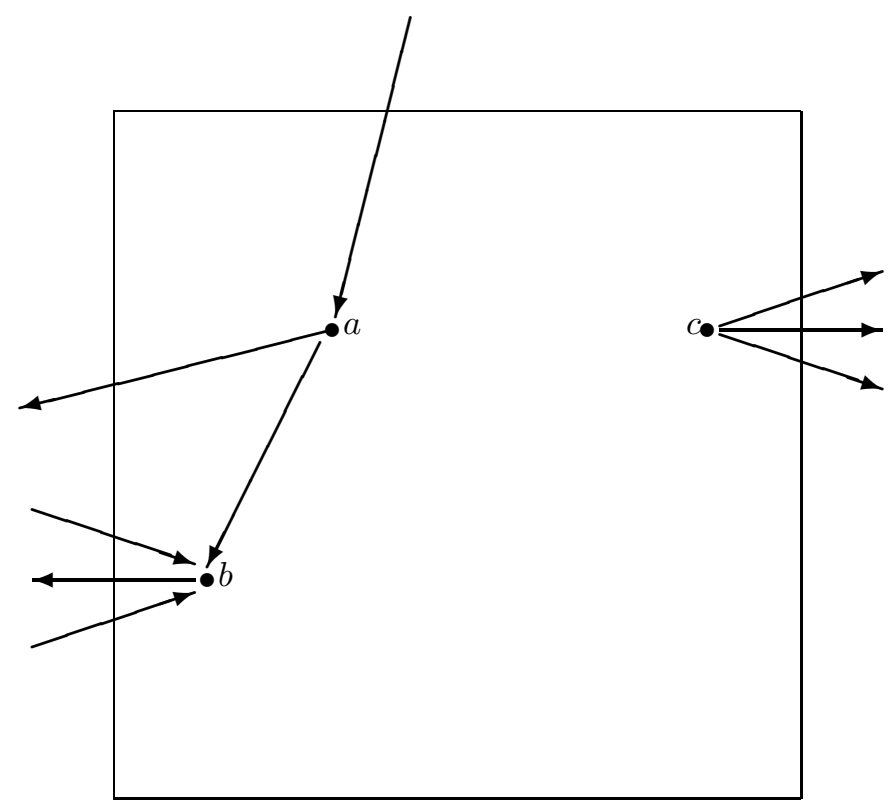

Figure 4. Figures $4-6$ build up the graph $\mathcal{G}_{\infty}^{*}\left(t_{+}\right)$on the time- $t_{+}$PWIT in Figure 1. Figure 4 here shows only the edges that were present at time $t_{-}$, that is the edges shown in Figure 3. Some edges crossing outside the window have been redrawn at different angles for later convenience.

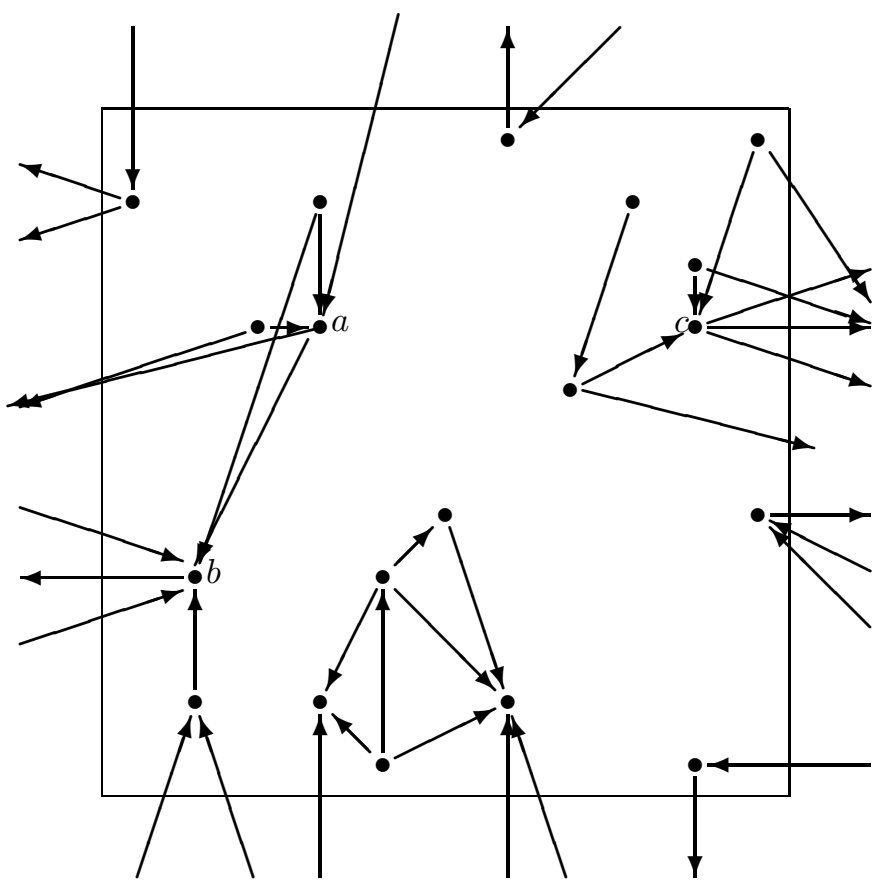

Figure 5. Suppose in Figure 1 that the center vertex $\emptyset$ arrives at time $t_{+}$; Figure 5 illustrates the graph just before that arrival. Since the time- $t_{-}$configuration in Figure 3 , more vertices have arrived and formed edges, and distances have expanded. 


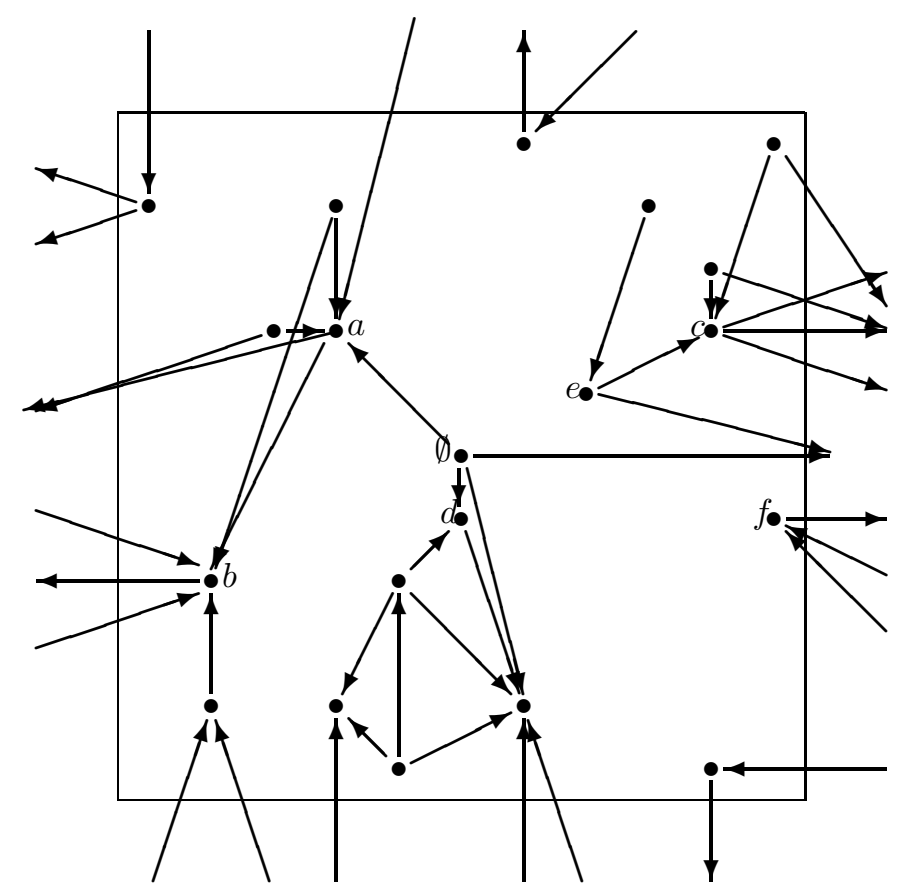

Figure 6. Vertex $\emptyset$ arrives at time $t_{+}$with near neighbors $d, e, a, f, \ldots$ Out-edges from $\emptyset$ appear according to the metric copying scheme of section 3.1 with $p(x)$ given by (23). So for each near neighbor $v$ and each existing edge $(v, w)$, an edge $(\emptyset, v)$ or $(\emptyset, w)$ appears with probability $p(D(\emptyset, v))$. In this realization, edges appeared to the near neighbors $d$ and $a$, and two other edges (one from $d$ and one from $e$ ) were copied.

Our focus in this paper is the study of the MFSC model using its limit structure. Just as the $t \rightarrow \infty$ limit of the time- $t$ "geometry" $\mathcal{D}_{t}$ is the PWIT, we can consider $\mathcal{G}_{t}$ as a structure built over $\mathcal{D}_{t}$, and we get a limit random directed graph $\mathcal{G}_{\infty}^{*}$ as a structure built over the PWIT. The structure of $\mathcal{G}_{\infty}^{*}$ near the root is exactly the $t \rightarrow \infty$ limit structure of $\mathcal{G}_{t}$ relative to a random ("typical") vertex $V_{t}$, and so we can obtain (in principle) a wide variety of asymptotic results about $\mathcal{G}_{t}$ by doing calculations upon $\mathcal{G}_{\infty}^{*}$. The only difficulty in this methodology is that we don't have a useful explicit description of $\mathcal{G}_{\infty}^{*}$. Instead, we can consider the space-time limits (2021) jointly with $\left(\mathcal{G}_{t}\right)$; then in the limit we get the space-time PWIT processes existing jointly with a random graph process $\left(\mathcal{G}_{\infty}^{*}(s)\right)$, where now $\mathcal{G}_{\infty}^{*}(0)=\mathcal{G}_{\infty}^{*}$. The process $\left(\mathcal{G}_{\infty}^{*}(s)\right)$ evolves with $s$ and the space-time PWIT process by the rules implied by (i)-(iii) above.

Precisely, the evolution rules (illustrated by Figures $3-6$ ) are

when a new vertex $v^{*}$ arrives in the forwards space-time PWIT process at time $s$, it has near neighbors $\left(v_{1}, v_{2}, \ldots\right)$ at distances $\left(\xi_{1}, \xi_{2}, \ldots\right)$, and $\mathcal{G}_{\infty}^{*}(s)$ has put a random graph structure on the geometric component containing each $v_{i}$. For each $i$ and each directed edge $\left(v_{i}, w\right)$, a new edge $\left(v^{*}, v_{i}\right)$ or $\left(v^{*}, w\right)$ is created with probability $p\left(D\left(v^{*}, v_{i}\right)\right)$, independently for different possible edges. 
Recursive self-similarity. What makes this process tractable is that the recursive self-similarity property of the PWIT extends to the random graph process; each $v_{i}$ defines a geometric component and a random graph on that component, and these are independent copies of the joint distribution of the PWIT and $\mathcal{G}_{\infty}^{*}$. This property is used extensively in the calculations in section 4

\subsection{Reparametrization and extreme cases}

Although the MFSC model makes sense for the full range $(0<\alpha, \lambda<\infty)$ of parameters, we will only consider the ranges (45) for which the limit mean degree is finite.

Note that definition (23) of $p(x)$ can be rewritten as

$$
\begin{aligned}
& p(x)=\alpha \lambda e^{-\lambda x}, 0<x<\infty \quad[\text { low }] \\
& p(x)=\left\{\begin{array}{ll}
1 & 0<x \leq \eta \\
e^{-\lambda(x-\eta)} & \eta<x<\infty
\end{array} \quad[\text { high }]\right.
\end{aligned}
$$

where the reparametrization $\eta:=\lambda^{-1} \log (\alpha \lambda)$ when $\alpha \lambda>1$ is used in (25). In the two extremes of clustering, our model simplifies in different ways. For fixed $\alpha$, when $n$ is large and $\alpha$ is small, the model resembles the following variant of the proportional attachment model:

An arriving vertex has a $\operatorname{Poi}(\alpha)$ number of out-edges, whose endvertices are chosen with probabilities proportional to $1+$ in-degree.

At the other extreme, for fixed $\eta$ our model makes sense with $\lambda=\infty$, interpreting (25) to mean $p(x)=0, x>\eta$. In this case the model becomes, for large $n$,

An arriving vertex $v^{*}$ chooses at random a Poi $(\eta)$ number of neighbors $v_{j}$, and creates edges $\left(v^{*}, v_{j}\right)$, and also copies each existing edge $\left(v_{j}, w\right)$ to a new edge $\left(v^{*}, w\right)$.

Clearly in the former limit we have $\kappa_{\text {cluster }}=0$ and in the latter limit we have $\kappa_{\text {cluster }}=1$.

\section{Calculations}

In section 4 we derive the formulas stated in sections 2.2 - 2.6 As described in section 3.5 our methodology is to regard $D_{\text {in }}$ and $D_{\text {out }}$ as the (random) in-degree and out-degree of the root in $\mathcal{G}_{\infty}^{*}$, and to study this using the timedynamics of $\mathcal{G}_{\infty}^{*}(s)$ derived from the space-time PWIT process and the evolution rules of the graph process. 


\subsection{Two helpful calculations}

We will make frequent use of the next lemma.

Lemma 1 For integers $u \geq 1$ write

$$
Z^{(u)}=\sum_{i=1}^{\infty} p^{u}\left(\xi_{i}\right)=\sum_{i=1}^{\infty}\left[\min \left(1, \alpha \lambda e^{-\lambda \xi_{i}}\right)\right]^{u} .
$$

Then

$$
\begin{aligned}
& \beta_{u}:=E Z^{(u)}=\left\{\begin{array}{cc}
u^{-1} \alpha^{u} \lambda^{u-1} & \text { [low }] \\
\eta+\frac{1}{u \lambda} & \text { [high] }
\end{array}\right. \\
& \operatorname{var} Z^{(1)}=\left\{\begin{array}{cc}
\frac{1}{2} \alpha^{2} \lambda & \text { [low }] \\
\eta+\frac{1}{2 \lambda} & \text { [high]. }
\end{array}\right.
\end{aligned}
$$

In particular, $\beta:=\beta_{1}<1$ for all parameter values.

Proof. We will do the low clustering density case - the high density case is similar. By (3) the chance that some $\xi_{i}$ falls into an interval $[x, x+d x]$ is $d x$, so

$$
\beta_{u}=\int_{0}^{\infty}\left(\alpha \lambda e^{-\lambda x}\right)^{u} d x=\alpha^{u} \lambda^{u} \int_{0}^{\infty} e^{-u \lambda x} d x=\alpha^{u} \lambda^{u} /(u \lambda) .
$$

Moreover, there is a general formula for variance of a sum over a Poisson (rate 1) process $\left(\xi_{i}\right)$ :

$$
\operatorname{var}\left(\sum_{i} w\left(\xi_{i}\right)\right)=\int_{0}^{\infty} w^{2}(x) d x
$$

and applying this formula for $Z^{(1)}$ gives

$$
\operatorname{var} Z^{(1)}=\int_{0}^{\infty}\left(\alpha \lambda e^{-\lambda x}\right)^{2} d x=\alpha^{2} \lambda^{2} /(2 \lambda) .
$$

We next recall a classical result. Fix $0<\theta<\infty$. Set $N(0)=1$ and let $(N(t), t \geq 0)$ be the Yule process of rate $\theta$, that is the Markov process which changes only by +1 steps and for which

$$
P(N(t+d t)=n+1 \mid N(t)=n)=\theta n d t .
$$

A textbook result (e.g. [18] sec. 5.3) says

$$
N(t) \stackrel{d}{=} \operatorname{Geo}\left(e^{-\theta t}\right) .
$$

Note that in the PWIT, if $N(r)$ is the number of vertices within distance $r$ from the root (counting the root itself), then the process $(N(r), r \geq 0)$ is a Yule process of rate 1 , because for a vertex $v$ at distance $r^{\prime}<r$, the chance of $v$ having a near neighbor at distance $\in\left[r-r^{\prime}, r-r^{\prime}+d r\right]$ equals $1 \cdot d r$. So in particular,

$$
E N(r)=E \operatorname{Geo}\left(e^{-r}\right)=e^{r}
$$




\subsection{Distribution of in-degree}

We start by giving the derivation of

$$
1+D_{\text {in }} \stackrel{d}{=} \operatorname{Geo}\left(e^{-\beta T}\right) \text { where } T \stackrel{d}{=} \operatorname{Exp}(1)
$$

for $\beta=E Z^{(1)}$. In the forwards space-time PWIT process, let $N(t)$ be $1+$ the in-degree of the root, when the root has age $t$. Thus $N(t)$ counts the set of vertices $v$ for which $v \rightarrow$ root is an edge, or $v=$ root. When a new vertex $v^{\prime}$ arrives with some $v$ in this set as a near neighbor, at distance $r$, there is chance $p(r)$ for the root's in-degree to increase by 1 , and so from the dynamics (22) of the forwards space-time PWIT process we see that $N(t)$ is the Yule process of rate

$$
\beta=\int_{0}^{\infty} p(r) d r
$$

Use formula (28) and the fact that the age of the root of the PWIT has $\operatorname{Exp}(1)$ distribution to obtain (8).

We can quickly use (8) to calculate $E D_{\text {in }}$.

$$
\begin{aligned}
1+E D_{\text {in }} & =E\left(E\left(\operatorname{Geo}\left(e^{-\beta T}\right) \mid T\right)\right) \\
& =E e^{\beta T} \text { because } E \operatorname{Geo}(p)=p^{-1} \\
& =\int_{0}^{\infty} e^{\beta t} e^{-t} d t=\frac{1}{1-\beta}
\end{aligned}
$$

giving $E D_{\text {in }}=\frac{\beta}{1-\beta}$ as at (6). We now calculate the distribution of $D_{\text {in }}$ in the same way. Because $P(\operatorname{Geo}(p) \geq i+1)=(1-p)^{i}, i \geq 0$ we have

$$
\begin{aligned}
P\left(D_{\text {in }} \geq i\right) & =E P\left(D_{\text {in }} \geq i \mid T\right) \\
& =E P\left(1+D_{\text {in }} \geq i+1 \mid T\right) \\
& =E\left(1-e^{-\beta T}\right)^{i} \\
& =\int_{0}^{\infty}\left(1-e^{-\beta t}\right)^{i} e^{-t} d t \\
& =\frac{1}{\beta} \int_{0}^{1}(1-s)^{i} s^{\frac{1}{\beta}-1} d s \text { setting } s=e^{-\beta t} \\
& =\frac{1}{\beta} \frac{\Gamma(i+1) \Gamma\left(\frac{1}{\beta}\right)}{\Gamma\left(i+1+\frac{1}{\beta}\right)} \text { using the Beta integral formula. }
\end{aligned}
$$

This is (10), and (11) follows.

Historical note. Yule [20] introduced what we now call the Yule process in 1924 in the context of a model for evolution of new species. It is interesting that his central mathematical results are the Geometric distribution (28) [his (5)] and the calculation starting from our (8) [representing, for Yule, a distribution of numbers of species in a typical genus] of the explicit distribution (11) [his (12)]. After 80 years we have slicker notation but the argument is the same! Moreover 
Yule's motivation was to find a simple model yielding a power-law distribution for number of species per genus, just as the motivation for the recent literature on proportional attachment models was to find a simple model yielding powerlaw degree distributions.

\subsection{Distribution of out-degree}

We will first derive (12). Because the out-edges are formed on arrival, we may suppose the root of the PWIT has just arrived. Consider a near neighbor $v^{\prime}$ at distance $r$. For each out-edge of $v^{\prime}$, and for $v^{\prime}$ itself, there is chance $p(r)$ that a corresponding out-edge is created at the root, giving a total number $\operatorname{Bin}\left(1+D\left(v^{\prime}\right), p(r)\right)$ of out-edges, where $D\left(v^{\prime}\right)$ is the out-degree of $v^{\prime}$. The recursive self-similarity property (end of section 3.5) implies that the $\left(D\left(v^{\prime}\right)\right.$ : $v^{\prime}$ near neighbor of root) are i.i.d. random variables distributed as $D_{\text {out }}$, and independent of their distances $\left(\xi_{i}\right)$ from the root. Rewriting $\left(D\left(v^{\prime}\right)\right)$ as $\left(D^{(i)}\right)$ in increasing order of distance from root,

$$
D_{\text {out }}=\sum_{i=1}^{\infty} \operatorname{Bin}\left(1+D^{(i)}, p\left(\xi_{i}\right)\right)
$$

which becomes (12).

We now turn to the issue of using (30) to get information about the distribution of $D_{\text {out }}$. Because a directed edge contributes equally to total in-degree and to total out-degree, we know a priori that $E D_{\text {out }}$ must equal $E D_{\text {in }}$, but let us first check that we can indeed use (12) to show $E D_{\text {out }}=\beta /(1-\beta)$. Because (11) $E \operatorname{Bin}(n, p)=n p$ we see

$$
E \operatorname{Bin}\left(1+D_{i}, p\left(\xi_{i}\right)\right)=\left(1+E D_{\text {out }}\right) E p\left(\xi_{i}\right) .
$$

So (30) gives

$$
E D_{\text {out }}=\left(1+E D_{\text {out }}\right) \cdot E Z^{(1)}=\left(1+E D_{\text {out }}\right) \beta
$$

giving $E D_{\text {out }}=\beta /(1-\beta)$.

Variance. The calculation of the variance var $D_{\text {out }}$ provides a textbook illustration of the utility of the general conditional variance formula

$$
\operatorname{var} X=E \operatorname{var}(X \mid Y)+\operatorname{var} E(X \mid Y) \text {. }
$$

We give the details in the low density case; the high density case is similar. In the defining equation (12) write $D$ for $D_{\text {out }}$ and write $\mathbf{D}$ and $\Xi$ for the random sequences $\left(D_{i}\right)$ and $\left(\xi_{i}\right)$. Because (2) var $\operatorname{Bin}(n, p)=n p(1-p)$ we have

$$
\operatorname{var}(D \mid \mathbf{D}, \Xi)=\sum_{i}\left(1+D_{i}\right) \alpha \lambda e^{-\lambda \xi_{i}}\left(1-\alpha \lambda e^{-\lambda \xi_{i}}\right) .
$$

Recursive self-similarity, as used above, implies independence of the i.i.d. sequence $\left(D_{i}, i \geq 1\right)$ and the Poisson process $\left(\xi_{i}, i \geq 1\right)$. So

$$
\begin{aligned}
E \operatorname{var}(D \mid \mathbf{D}, \Xi) & =(1+E D)\left(E Z^{(1)}-E Z^{(2)}\right) \\
& =\frac{\alpha\left(1-\frac{\alpha \lambda}{2}\right)}{1-\alpha} \text { using (6) and (26). }
\end{aligned}
$$


Next consider the conditional expectation

$$
E(D \mid \mathbf{D}, \Xi)=\sum_{i}\left(1+D_{i}\right) \alpha \lambda e^{-\lambda \xi_{i}}=W, \quad \text { say. }
$$

We will calculate var $W$ by using the conditional variance formula. Because

$$
\operatorname{var}(W \mid \Xi)=\sum_{i}(\operatorname{var} D) \cdot \alpha^{2} \lambda^{2} e^{-2 \lambda \xi_{i}}
$$

we have

$$
E \operatorname{var}(W \mid \Xi)=(\operatorname{var} D) \cdot E Z^{(2)}=(\operatorname{var} D) \cdot \alpha^{2} \lambda / 2 .
$$

And since $E(W \mid \Xi)=(1+E D) Z^{(1)}=\frac{1}{1-\alpha} Z^{(1)}$ we have

$$
\operatorname{var} E(W \mid \Xi)=\frac{1}{(1-\alpha)^{2}} \operatorname{var} Z^{(1)}=\frac{\alpha^{2} \lambda}{2(1-\alpha)^{2}} .
$$

Using the conditional variance formula twice

$$
\begin{aligned}
\operatorname{var} D & =E \operatorname{var}(D \mid \mathbf{D}, \Xi)+\operatorname{var} W \\
& =E \operatorname{var}(D \mid \mathbf{D}, \Xi)+E \operatorname{var}(W \mid \Xi)+\operatorname{var} E(W \mid \Xi) \\
& =\frac{\alpha\left(1-\frac{\alpha \lambda}{2}\right)}{1-\alpha}+(\operatorname{var} D) \cdot \alpha^{2} \lambda / 2+\frac{\alpha^{2} \lambda}{2(1-\alpha)^{2}} .
\end{aligned}
$$

Solving gives the equation (13) for var $D_{\text {out }}$.

Special cases. (a). Fix $\alpha$. Because $p(x) \leq \alpha \lambda$, in the $\lambda \rightarrow 0$ limit we can apply the Poisson limit of Binomials result to the defining equation (12) to obtain (cf. section 3.6)

$$
\text { (in } \lambda \rightarrow 0 \text { limit) } \quad D_{\text {out }} \stackrel{d}{=} \operatorname{Poi}(\alpha) .
$$

(b). Fix $\eta$. In the $\lambda \rightarrow \infty$ limit we can use the limit process of section 3.6 to show that $1+D_{\text {out }}$ has the distribution of the total population size in a Galton-Watson branching process ${ }^{6}$ with $\operatorname{Poi}(\eta)$ offspring distribution. This is (see e.g. [5]) the Borel-Tanner $(\eta)$ distribution

$$
\text { (in } \lambda \rightarrow \infty \text { limit) } \quad P\left(1+D_{\text {out }}=d\right)=\frac{(\eta d)^{d-1} e^{-\eta d}}{d !}, \quad d \geq 1 .
$$

(c). In the case $\alpha \lambda=1$ it turns out (an argument is sketched in section [5.4)

$$
1+D_{\text {out }} \stackrel{d}{=} \operatorname{Geo}(1-\alpha) .
$$

Independence of in-degree and out-degree. This independence, noted at (14), follows from the fact that in the forwards space-time PWIT process the outdegree of the root is determined at the arrival time of the root vertex; the subsequent evolution of the process of in-edges is clearly independent of the state of the graph immediately after arrival.

\footnotetext{
${ }^{6} \mathrm{~A}$ population process starting with one individual in generation 0 , individuals having i.i.d. random numbers of offspring in successive generations
} 


\subsection{Densities of induced subgraphs}

Here we give details of the definition and interpretation of "density of induced subgraph" mentioned in section 2.4 and list explicit formulas.

Let $G$ and $\mathcal{G}$ be finite directed acyclic graphs; think of $G$ as small and $\mathcal{G}$ as large. Define "density of $G$ as an induced subgraph of $\mathcal{G}$ " by

$$
\operatorname{dens}(G \mid \mathcal{G})=\frac{\#\{V \subset \mathcal{G}: V \text { isomorphic to } G\}}{\#\{\text { vertices of } \mathcal{G}\}}
$$

where \# denotes cardinality ("number of") and $V$ denotes a vertex-subset of $\mathcal{G}$ with its induced subgraph. See Figure 7, where there are 3 such vertex-subsets $\{a, b, e\},\{b, c, e\},\{c, d, e\}$ and so where $\operatorname{dens}(G \mid \mathcal{G})=3 / 5$.
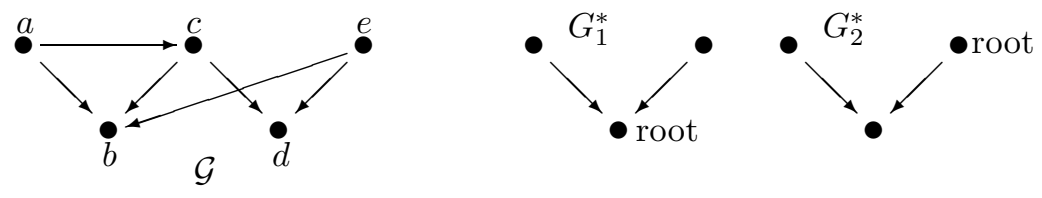

Figure 7. Two rootings $G_{1}^{*}, G_{2}^{*}$ of a graph $G$.

We want to study $n \rightarrow \infty$ limits of $\operatorname{dens}\left(G \mid \mathcal{G}_{n}\right)$ in our MFSC random graph model $\left(\mathcal{G}_{n}\right)$, for fixed $G$. To use our methodology we must first rephrase the definition of $\operatorname{dens}(G \mid \mathcal{G})$ in terms of the rooted graph $\mathcal{G}^{*}$ obtained by giving $\mathcal{G}$ a uniform random root. For such $\mathcal{G}^{*}$, and for an arbitrarily-rooted directed graph $G^{*}$, define a random variable

$\mathbf{X}\left(G^{*} \mid \mathcal{G}^{*}\right)=$ number of vertex-subsets $V$ of $\mathcal{G}^{*}$ including the root such that $\mathcal{G}^{*}$ restricted to $V$ is isomorphic to $G^{*}$ via a root-preserving isomorphism.

The randomness arises only from choice of root of $\mathcal{G}^{*}$; notation $\mathbf{X}$ has no special significance except to distinguish this from simpler random variables.

It is an easy fact that

$$
\operatorname{dens}(G \mid \mathcal{G})=\frac{E \mathbf{X}\left(G^{*} \mid \mathcal{G}^{*}\right)}{\iota\left(G^{*}\right)}
$$

where $G^{*}$ is $G$ with an arbitrary choice of root, and where $\iota\left(G^{*}\right) \geq 1$ is the number of different root-choices which would give a rooted graph isomorphic to this particular choice. Rather than write a formal proof, let us just illustrate identity (37) using Figure 7. For the choice of root giving $G_{1}^{*}$, the number of isomorphic vertex-subsets $V$ of $\mathcal{G}^{*}$ equals 2 (resp. 1) if the root of $\mathcal{G}^{*}$ chances to be $b$ (resp. $d$ ), and so $E \mathbf{X}\left(G_{1}^{*} \mid \mathcal{G}^{*}\right)=3 / 5$. For the choice of root giving $G_{2}^{*}$, the number of isomorphic vertex-subsets $V$ of $\mathcal{G}^{*}$ equals 1 (resp. 2,3) if the root of $\mathcal{G}^{*}$ chances to be $a$ (resp. $\left.c, e\right)$, and so $E \mathbf{X}\left(G_{2}^{*} \mid \mathcal{G}^{*}\right)=6 / 5$. Since $\iota\left(G_{1}^{*}\right)=1$ while $\iota\left(G_{2}^{*}\right)=2$, we have checked identity (37) in this example.

Note that in Figure 7, the induced subgraph on $\{a, b, c\}$ is not isomorphic to $G$ because of the extra edge $a \rightarrow c$. Obviously we can make parallel definitions 
allowing extra edges (pedantically: replace "isomorphism" by "vertex-bijection and edge-surjection") and we write dens $(G \mid \mathcal{G})$ and $\overline{\mathbf{X}}\left(G^{*} \mid \mathcal{G}^{*}\right)$ in this setting. For instance, in Figure 7 we have dens $(G \mid \mathcal{G})=4 / 5$ and $E \overline{\mathbf{X}}\left(G_{1}^{*} \mid \mathcal{G}^{*}\right)=4 / 5$.

The point of all this is that the definition of $\mathbf{X}\left(G^{*} \mid \mathcal{G}^{*}\right)$ makes sense when $\mathcal{G}^{*}$ is a rooted infinite graph. The key methodology in our analysis of the MFSC model $\left(\mathcal{G}_{n}\right)$ is that the randomly-rooted $\mathcal{G}_{n}^{*}$ converge locally to a limit random infinite rooted graph $\mathcal{G}_{\infty}^{*}$, implying via (37) that

$$
\operatorname{dens}\left(G \mid \mathcal{G}_{n}\right) \rightarrow \frac{E \mathbf{X}\left(G^{*} \mid \mathcal{G}_{\infty}^{*}\right)}{\iota\left(G^{*}\right)}:=\operatorname{dens}_{\infty}(G), \text { say. }
$$

In parallel.

$$
\overline{\operatorname{dens}}\left(G \mid \mathcal{G}_{n}\right) \rightarrow \frac{E \overline{\mathbf{X}}\left(G^{*} \mid \mathcal{G}_{\infty}^{*}\right)}{\iota\left(G^{*}\right)}:=\overline{\operatorname{dens}_{\infty}}(G), \text { say. }
$$

In sections 4.5 and 5.2 we calculate $\operatorname{dens}(G)$ for several cases of $G$; let us record the formulas below. Note that limit densities may be infinite, in which cases we will point out the conditions on parameters needed for finiteness.

(a). For a directed path $\pi_{r}$ with $r \geq 1$ edges,

$$
\overline{\operatorname{dens}}_{\infty}\left(\pi_{r}\right)=\partial^{r} .
$$

(b). For the complete directed acyclic graph $K_{r}$ on $r \geq 2$ vertices, (that is, vertices $\{1,2, \ldots, r\}$ and edges $i \rightarrow j$ for $1 \leq i<j \leq r$ ),

$$
\operatorname{dens}_{\infty}\left(K_{r}\right)=\prod_{u=1}^{r-1} \frac{\beta_{u}}{1-\beta_{u}}
$$

where $\beta_{1}=\beta$ and for general $u \geq 1$

$$
\beta_{u}:=\left\{\begin{array}{cc}
u^{-1} \alpha^{u} \lambda^{u-1} & {[\text { low }]} \\
\eta+\frac{1}{u \lambda} & {[\text { high }] .}
\end{array}\right.
$$

In particular, for the case of triangles $K_{3}$ we have explicitly

$$
\operatorname{dens}_{\infty}\left(K_{3}\right)= \begin{cases}\frac{\alpha^{3} \lambda}{(1-\alpha)\left(2-\alpha^{2} \lambda\right)} & {[\text { low }]} \\ \frac{\left(\eta+\frac{1}{\lambda}\right)\left(\eta+\frac{1}{2 \lambda}\right)}{\left(1-\eta-\frac{1}{\lambda}\right)\left(1-\eta-\frac{1}{2 \lambda}\right)} & {[\text { high }] .}\end{cases}
$$

As already mentioned in section 2.4 the formula above is the key ingredient in the formula for $\kappa_{\text {cluster }}$. Recall its verbal description

The proportion of directed 2-paths $v_{1} \rightarrow v_{2} \rightarrow v_{3}$ for which $v_{1} \rightarrow v_{3}$ is also an edge.

This becomes

$$
\kappa_{\text {cluster }}=\frac{\operatorname{dens}_{\infty}\left(K_{3}\right)}{\overline{\operatorname{dens}}{ }_{\infty}\left(\pi_{2}\right)}
$$


and then (4044) immediately give the formula (7).

(c). For a directed path $\pi_{r}$ with $r \geq 1$ edges,

$$
\operatorname{dens}_{\infty}\left(\pi_{r}\right)=\delta\left(\frac{\beta_{1}-\beta_{2}}{\left(1-\beta_{1}\right)\left(1-\beta_{2}\right)}\right)^{r-1} .
$$

(d). For the complete bipartite directed graph $K_{2,2}$, for $\beta_{2}<\frac{1}{2}$ (which always holds in the low density case)

$$
\overline{\operatorname{dens}}_{\infty}\left(K_{2,2}\right)=\frac{\partial \beta_{2}\left(\beta_{2}+\frac{1}{2} \partial \beta\right)}{\left(1-2 \beta_{2}\right)\left(1-\beta_{2}\right)} .
$$

(e). In principle one can calculate $\operatorname{dens}_{\infty}\left(G^{*}\right)$ for any $G^{*}$, but in practice it is not clear to what extent useful explicit formulas can be found - see section 4.5 for further discussion, and for the observation that certain graphs $G^{*}$ have $\operatorname{dens}_{\infty}\left(G^{*}\right)=0$, "asymptotically negligible density".

\subsection{Densities of induced subgraphs: easy explicit formulas}

Recall the basic result (38) on subgraph density:

$$
\operatorname{dens}\left(G \mid \mathcal{G}_{n}\right) \rightarrow \frac{E \mathbf{X}\left(G^{*} \mid \mathcal{G}_{\infty}^{*}\right)}{\iota\left(G^{*}\right)}:=\operatorname{dens}_{\infty}(G)
$$

where $G^{*}$ is an arbitrary rooting of $G$. In calculating the right side, to simplify notation we write

$$
\chi\left(G^{*}\right)=E \mathbf{X}\left(G^{*} \mid \mathcal{G}_{\infty}^{*}\right)
$$

and similarly for $\bar{\chi}(\cdot)$ and $\overline{\mathbf{X}}(\cdot)$ and $\overline{\mathrm{dens}}(G)$.

First consider $\pi_{r}$, the directed path with $r$ edges, rooted at the last-arriving vertex, which we will call the head. Clearly $\chi\left(\pi_{1}\right)=E D_{\text {out }}=\partial$. Let us write out the (rather obvious) inductive argument for calculating $\bar{\chi}\left(\pi_{r}\right)$. Whether or not the root vertex of $\mathcal{G}_{\infty}^{*}$ is the head of a $r$-path is determined at its arrival time. Consider a near neighbor $v_{i}$ of the root, at distance $\xi_{i}$. The expected number of $r-1$-paths headed by $v_{i}$ equals $\bar{\chi}\left(\pi_{r-1}\right)$. So the expected number of $r$ paths of the form root $\rightarrow v_{i} \rightarrow \ldots$ equals $\bar{\chi}\left(\pi_{r-1}\right) \times P\left(\left(\right.\right.$ root, $\left.v_{i}\right)$ is edge of $\left.\mathcal{G}_{\infty}^{*}\right)$. Summing over $i$ gives

$$
\bar{\chi}\left(\pi_{r}\right)=\bar{\chi}\left(\pi_{r-1}\right) \times E D_{\text {out }}=\bar{\chi}\left(\pi_{r-1}\right) \times \partial
$$

and so $\bar{\chi}\left(\pi_{r}\right)=\partial^{r}$ by induction. This is formula (40).

The result for the complete directed graph $K_{r}$ on $r$ vertices is similar. For $r=2$ we have $\chi\left(K_{2}\right)=\chi\left(\pi_{1}\right)=\partial$ and so to establish formula (41) by induction it is enough to show

$$
\chi\left(K_{r+1}\right)=\chi\left(K_{r}\right) \times \frac{\beta_{r}}{1-\beta_{r}} .
$$

In the forwards space-time PWIT process, consider a vertex-set $S_{r}$ isomorphic to $K_{r}$, headed by its latest-arriving vertex $v_{*}$. At time $t$ after the arrival of $v_{*}$, 
let $N_{t}=1+$ the number of $K_{r+1}$-subgraphs of the forwards space-time PWIT process which are of the form $\{v\} \cup S_{r}$ for some $v$; regard the " +1 " as counting $S_{r}$ itself. Then $N_{t}$ is a Yule process of rate

$$
E \sum_{i} p^{r}\left(\xi_{i}\right)=\beta_{r}
$$

because for each vertex $v$ counted in $N_{t}$, a new vertex $v^{\prime}$ arriving with near neighbor $v$ at distance $x$ has chance $[p(x)]^{r}$ to create the $r$ edges needed to make $\left\{v^{\prime}\right\} \cup S_{r}$ be a $K_{r+1}$ subgraph. Moreover these are the only ways in which a new $K_{r+1}$ of the form $\left\{v^{\prime}\right\} \cup S_{r}$ can be formed. By the Yule formula (29) $N_{t} \stackrel{d}{=} \mathrm{Geo}\left(\exp \left(-t \beta_{r}\right)\right)$. Now regard $K_{r+1}$ as rooted by its second-latest arriving vertex. In $\mathcal{G}_{\infty}^{*}$ the root has age $T \stackrel{d}{=} \operatorname{Exp}(1)$. At its arrival time the root headed some random number of $K_{r}$ 's, with mean $\chi\left(K_{r}\right)$, so by considering the mean number of $K_{r+1}$ 's at the present time

$$
\begin{aligned}
\chi\left(K_{r+1}\right) & =\chi\left(K_{r}\right) \times\left(E N_{T}-1\right) \\
& =\chi\left(K_{r}\right) \times\left(E \exp \left(T \beta_{r}\right)-1\right) \\
& =\chi\left(K_{r}\right) \times\left(\frac{1}{1-\beta_{r}}-1\right)
\end{aligned}
$$

giving (47).

Other subgraphs. The derivations of formulas (45146) dealing with paths and $K_{2,2}$ are relegated to sections 5.1 and 5.2

For the graph out $-\operatorname{star}_{r}$ consisting of $r$ out-edges at a root, it is clear that

$$
\bar{\chi}\left(\text { out }-\operatorname{star}_{r}\right)=E\left(\begin{array}{c}
D_{\text {out }} \\
r
\end{array}\right)
$$

and similarly

$$
\bar{\chi}\left(\text { in }-\operatorname{star}_{r}\right)=E\left(\begin{array}{c}
D_{\text {in }} \\
r
\end{array}\right),
$$

and these can in principle be evaluated using (128).

Some subgraphs have density zero.

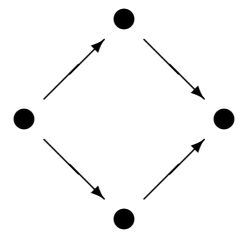

It is easy to see that the graph $G^{*}$ above (where no "vertical" edge is present) has $\chi\left(G^{*}\right)=0$.

\subsection{Reparametrization}

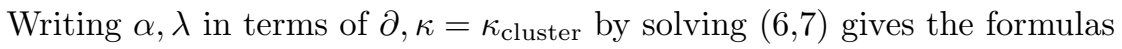

$$
\left.\begin{array}{l}
\alpha= \\
\lambda=\frac{\partial}{\partial+1} \\
\lambda\left(1+\frac{1}{\partial}\right)^{2} \\
1+\frac{1}{\partial \kappa}
\end{array}\right\} 0<\kappa \leq \frac{1}{\partial+2}
$$




$$
\left.\begin{array}{l}
\eta=\frac{\partial((\partial+2) \kappa-1)}{(\partial+1)(1+\partial \kappa)} \\
\lambda=\frac{1}{2 \partial(1-\kappa)}
\end{array}\right\} \frac{1}{\partial+2}<\kappa<1
$$

\subsection{Edge-lengths}

The previous calculations have not made very extensive use of the time-dynamics of the forwards space-time PWIT process, and in particular have not used the fact that edge-lengths grow exponentially at rate 1 . To derive the formula (17) for edge length density we do need to exploit such time-dynamics. We consider only the low-density case; the high density case is more complicated because the distribution in (ii) below is no longer exponential.

Consider the lengths of the in-edges at a particular vertex $v_{0}$. Following a tradition in mathematical probability, we visualize an in-edge of length $\ell$ as a "particle" at position $\ell$ on a line; we also put a particle at position 0 to represent the vertex $v_{0}$ itself. If we start time $\tau$ with $\tau=0$ at the arrival time of $v_{0}$, then the evolution of the "particle process" can be specified as follows.

(i) There is a particle at position 0 at all times $\tau \geq 0$.

(ii) For each particle (at position $x$ at time $\tau$, say), at stochastic rate $\alpha$ per unit time a new particle appears at position $x+\operatorname{Exp}(\lambda)$.

(iii) particle positions increase deterministically at exponential(1) rate: a particle at $x$ at time $\tau$ will be at $x e^{\tau_{0}-\tau}$ at time $\tau_{0}>\tau$.

Rule (ii) derives from (22): for an existing edge $\left(v^{\prime}, v_{0}\right)$, a new vertex arriving at distance $r$ from near neighbor $v^{\prime}$ creates an edge to $v_{0}$ with probability $p(r)$, so the rate at which each existing edge is copied equals $\int_{0}^{\infty} p(x) d x=\alpha$; moreover conditional on copying, the distance $r$ has $\operatorname{Exp}(\lambda)$ distribution, and so the length of the new edge equals the length of the old edge $+\operatorname{Exp}(\lambda)$.

To analyze this particle process of edge lengths, define

$$
G(\tau, x)=E \text { (number of edges of length }>x \text { at time } \tau \text { ) }
$$

so that

$$
g(\tau, x)=-\frac{d}{d x} G(\tau, x)=\text { mean edge-length density at time } \tau .
$$

We shall study

$f(x) d x=E$ (number of in-edges at a typical vertex with length $\in[x, x+d x]$ ).

Because the age of a typical vertex has $\operatorname{Exp}(1)$ distribution, $f(x)$ can be written as

$$
f(x)=\int_{0}^{\infty} g(\tau, x) e^{-\tau} d \tau .
$$

The verbal description of the particle process leads to the equation

$$
\frac{d}{d \tau} G(\tau, x)=x g(\tau, x)+\alpha \int_{0}^{x} g(\tau, y) e^{-\lambda(x-y)} d y+\alpha e^{-\lambda x} .
$$


Here the first term on the right expresses the deterministic exponential growth, the second term expresses birth of particles to parents not at 0 (copying of existing edges) and the third expresses births to the 0 -particle (new edge to $v_{0}$ ). Multiply the terms of the equation by $e^{-\tau}$ and integrate out $\tau$; noting

$$
\int_{0}^{\infty} \frac{d}{d \tau} G(\tau, x) e^{-\tau} d \tau=\int_{0}^{\infty} G(\tau, x) e^{-\tau} d \tau=F(x), \text { say }
$$

we obtain

$$
F(x)=x f(x)+\alpha \int_{0}^{x} f(y) e^{-\lambda(x-y)} d y+\alpha e^{-\lambda x} .
$$

Differentiate: $-f=(x f)^{\prime}+\alpha f-\lambda \alpha \int_{0}^{x} f(y) e^{-\lambda(x-y)} d y-\lambda \alpha e^{-\lambda x}$.

Rewrite with the integral term on the left, and then substitute the integral term by the expression implied in (51):

$$
\lambda\left(-x f-\alpha e^{-\lambda x}+F\right)=f+(x f)^{\prime}+\alpha f-\lambda \alpha e^{-\lambda x} .
$$

Differentiate: $\lambda\left(-(x f)^{\prime}+\lambda \alpha e^{-\lambda x}-f\right)=f^{\prime}+(x f)^{\prime \prime}+\alpha f^{\prime}+\lambda^{2} \alpha e^{-\lambda x}$.

Tidy: $(x f)^{\prime \prime}+\lambda(x f)^{\prime}+(1+\alpha) f^{\prime}+\lambda f=0$.

Look for a series solution $f(x)=\sum_{n=0}^{\infty} a_{n} x^{n}$. Equating coefficients of $x^{n}$ :

$$
(n+2)(n+1) a_{n+1}+\lambda(n+1) a_{n}+(1+\alpha)(n+1) a_{n+1}+\lambda a_{n}=0 .
$$

That is,

$$
\frac{a_{n+1}}{a_{n}}=\frac{-\lambda(n+2)}{(n+1)(n+3+\alpha)}
$$

and so

$$
a_{n}=\frac{(-\lambda)^{n}(n+1) \Gamma(3+\alpha)}{\Gamma(n+3+\alpha)} a_{0} .
$$

One can directly check that $f(0+)=1$, identifying $a_{0}=1$. Because the mean in-degree is $\alpha /(1-\alpha)$, the probability density function of a typical edge-length must be $\frac{1-\alpha}{\alpha} f(x)$, establishing (17).

\section{$5 \quad$ Further calculations}

\subsection{Yule arguments for subgraph density}

The next Lemma abstracts the Yule process arguments used in section 4.5 . Recall the reformulation there of limit subgraph density in terms of $\chi(G)$ and $\bar{\chi}(G)$. 

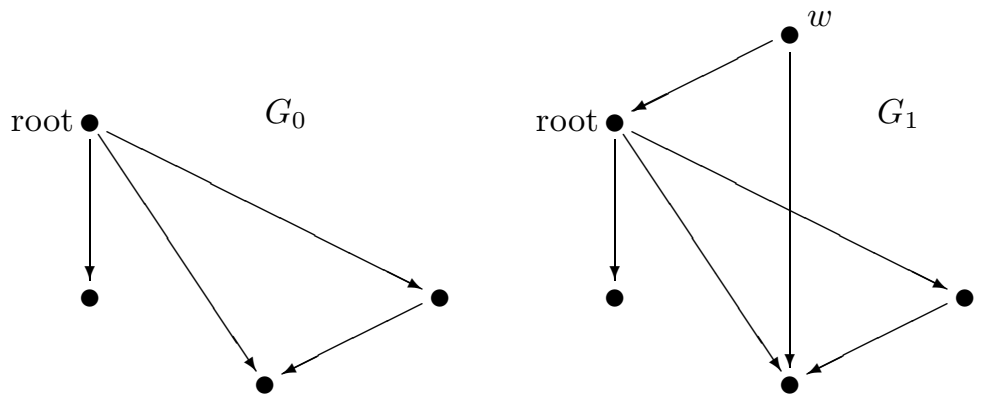

Figure 8. Illustration of Lemma 2

Lemma 2 Let $G_{0}$ be a rooted directed acyclic graph such that each vertex is a descendant of the root. Let $q$ be the out-degree of the root. Let $G_{1}$ be a directed acyclic graph obtained from $G_{0}$ by adding an extra vertex $w$ and edges ( $w$, root) and $c$ further edges from $w$ to some children of the root $($ so $0 \leq c \leq q)$. Then

$$
\begin{aligned}
\bar{\chi}\left(G_{1}\right) / \bar{\chi}\left(G_{0}\right) & =\frac{\beta_{c+1}}{1-\beta_{c+1}} . \\
\chi\left(G_{1}\right) / \chi\left(G_{0}\right) & =\sum_{j=0}^{q-c}(-1)^{j}\left(\begin{array}{c}
q-c \\
j
\end{array}\right) \frac{\beta_{c+1+j}}{1-\beta_{c+1+j}} .
\end{aligned}
$$

In particular, if $q=c$ then

$$
\chi\left(G_{1}\right) / \chi\left(G_{0}\right)=\frac{\beta_{c+1}}{1-\beta_{c+1}} .
$$

As a quick application let us derive formula [45) for $\chi\left(\pi_{r}\right)$ for the directed path $\pi_{r}$ on $r$ edges. Applying (53) with $q=1, c=0$,

$$
\chi\left(\pi_{r+1}\right) / \chi\left(\pi_{r}\right)=\frac{\beta_{1}}{1-\beta_{1}}-\frac{\beta_{2}}{1-\beta_{2}}=\frac{\beta_{1}-\beta_{2}}{\left(1-\beta_{1}\right)\left(1-\beta_{2}\right)} .
$$

Because $\chi\left(\pi_{1}\right)=\partial$ and $\iota\left(\pi_{r}\right)=1$ we obtain formula (45).

Proof of Lemma 2. We will do the harder case (53). Consider a copy of $G_{0}$ (i.e. an isomorphic subgraph) at the root of the PWIT. In the space-time PWIT process, let $M(t)$ be the number of copies of $G_{1}$ which contain the given copy of $G_{0}$, at time $t$ after the arrival of the root. Since the age $T$ of the root has $\operatorname{Exp}(1)$ distribution,

$$
\chi\left(G_{1}\right) / \chi\left(G_{0}\right)=E M(T) .
$$

Write $S$ for the set of children of the root in $G_{0}$ and write $A$ for a subset of $S$. 
Consider the process of arriving vertices $v$ which form an edge to the root. Such a $v$ has a near neighbor $v^{\prime}$, where either $v^{\prime}=$ root or $\left(v^{\prime}\right.$, root) is already an edge. Writing $A\left(v^{\prime}\right) \subseteq S$ for the set of children of the root to which $v^{\prime}$ creates an edge, then $A(v) \subseteq A\left(v^{\prime}\right)$. We can now write

$$
M(t)=\#\left\{v:(v, \text { root }) \text { is an edge, } A(v)=A_{1}\right\}
$$

where $A_{1}$ is the set of children of the root of $G_{1}$ to which $w$ has an edge. In representing $M(t)$ as above, we are using the hypothesis "each vertex is a descendant of the root" to ensure that, in a subgraph of the space-time PWIT isomorphic to $G_{1}$, the last-arriving vertex must be $w$.

Consider a sequence root $=v_{(0)}, v_{(1)}, \ldots, v_{(i)}$ of arriving vertices such that each vertex $v_{(k)}$ arrives at distance $x_{k}$ from its near neighbor $v_{(k-1)}$. The chance that each $v_{(k)}$ makes an edge to the root and to each child in $A_{1}$ equals $\prod_{k=1}^{i} p^{c+1}\left(x_{k}\right)$. The chance that furthermore no other child in $S$ acquires an edge to $v_{(i)}$ equals $\left(1-\prod_{k=1}^{i} p\left(x_{k}\right)\right)^{q-c}$. By considering the times $0<t_{1}<t_{2}<\ldots<t_{i}<t$ of arrivals of $v_{(i)}$,

$E M(t)=\sum_{i=1}^{\infty} \int_{0<t_{1}<\ldots<} \int_{t_{i}<t} d t_{1} \ldots d t_{i} \int_{0}^{\infty} \ldots \int_{0}^{\infty} d x_{1} \ldots d x_{i} \mathbf{p}^{c+1}(\mathbf{x})(1-\mathbf{p}(\mathbf{x}))^{q-c}$

where $\mathbf{p}(\mathbf{x})=\prod_{k=1}^{i} p\left(x_{i}\right)$. Because $\mathbf{p}^{c+1}(\mathbf{x})(1-\mathbf{p}(\mathbf{x}))^{q-c}=\sum_{j=0}^{q-c}(-1)^{j}\left(\begin{array}{c}q-c \\ j\end{array}\right) \mathbf{p}^{c+1+j}(\mathbf{x})$ and $\int_{0}^{\infty} p^{c+1+j}\left(x_{k}\right) d x_{k}=\beta_{c+1+j}$,

$$
\begin{aligned}
E M(t) & =\sum_{i=1}^{\infty} \frac{t^{i}}{i !} \sum_{j=0}^{q-c}(-1)^{j}\left(\begin{array}{c}
q-c \\
j
\end{array}\right) \beta_{c+1+j}^{i} \\
& =\sum_{j=0}^{q-c}(-1)^{j}\left(\begin{array}{c}
q-c \\
j
\end{array}\right)\left(\exp \left(\beta_{c+1+j} t\right)-1\right) .
\end{aligned}
$$

Calculating $\operatorname{EM}(T)=\int_{0}^{\infty} e^{-t} \operatorname{EM}(t) d t$ establishes (53).

\subsection{Subgraph density of $K_{2,2}$}

We have not pursued general methods for induced subgraph density beyond Lemma 2 but the argument that follows for the particular case of $K_{2,2}$, based on splitting into two cases, could clearly be applied somewhat more widely.

We first quote

\section{Lemma 3}

$$
\begin{aligned}
E\left(\begin{array}{c}
\operatorname{Geo}(p) \\
2
\end{array}\right) & =p^{-2}-p^{-1} \\
E\left(\begin{array}{c}
\operatorname{Geo}(p)-1 \\
2
\end{array}\right) & =p^{-2}-2 p^{-1}+1 .
\end{aligned}
$$


Next consider the graph $G^{*}$ on the left of Figure 9.
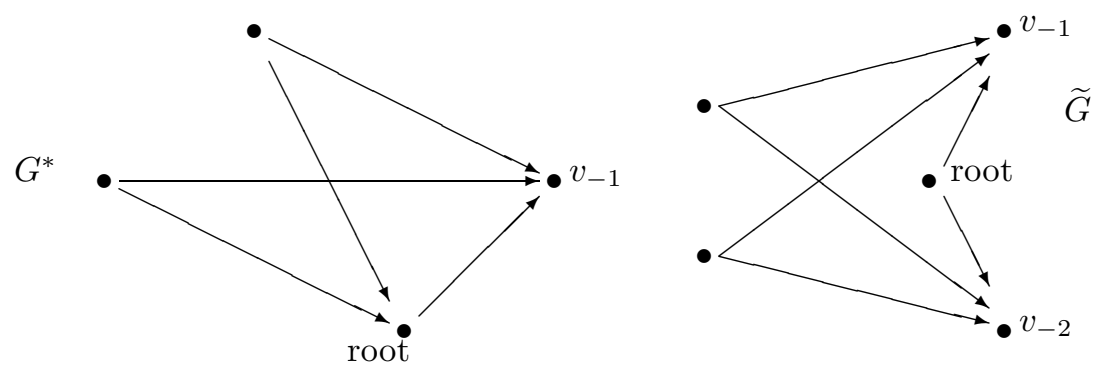

Figure 9. Graphs related to $K_{2,2}$.

We will show

$$
\bar{\chi}\left(G^{*}\right)=\frac{2 \beta_{2}^{2} \partial}{\left(1-2 \beta_{2}\right)\left(1-\beta_{2}\right)}
$$

where $\beta_{2}=E Z^{(2)}$.

We start by repeating the argument in the $r=2$ case of (47). In the forwards space-time PWIT process, consider the newly-arrived root and an edge (root, $v_{-1}$ ). At time $t$ after the arrival of the root, let $N_{t}=1+$ the number of vertices $v$ such that $\left(v\right.$,root) and $\left(v, v_{-1}\right)$ are both edges of the graph process; regard the " +1 " as counting the root itself. Then $N_{t}$ is a Yule process of rate $\beta_{2}=\int_{0}^{\infty} p^{2}(x) d x$. Thus at time $t$ there are $\left(\begin{array}{c}N_{t}-1 \\ 2\end{array}\right)$ graphs of the desired form containing the edge (root, $v_{-1}$ ). Because the age $T$ of the root has $\operatorname{Exp}(1)$ distribution, we see

$$
\bar{\chi}\left(G^{*}\right)=\partial E\left(\begin{array}{c}
N_{T}-1 \\
2
\end{array}\right)
$$

where $\partial=E D_{\text {out }}$ is the expected number of edges of the form (root, $v_{-1}$ ). Using (28) and (57),

$$
\begin{aligned}
\bar{\chi}\left(G^{*}\right) & =\partial E\left(e^{2 \beta_{2} T}-2 e^{\beta_{2} T}+1\right) \\
& =\partial\left(\frac{1}{1-2 \beta_{2}}-\frac{2}{1-\beta_{2}}+1\right)
\end{aligned}
$$

leading to (58).

Next, in $\mathcal{G}_{\infty}^{*}$ consider

$Q:=$ number of unordered pairs $\left(v_{-1}, v_{-2}\right)$ such that (root, $\left.v_{-1}\right)$ and (root, $v_{-2}$ ) are edges, and $v_{-1}$ and $v_{-2}$ were in different geometric components at the arrival time of the root.

By considering distances $r_{1}, r_{2}$ from the root to the near neighbors of the geometric components containing $v_{1}, v_{2}$,

$$
E Q=\frac{1}{2} \iint p\left(r_{1}\right) p\left(r_{2}\right) d r_{1} d r_{2} \times \partial^{2}=\beta^{2} \partial^{2} / 2 .
$$


Now consider in $\mathcal{G}_{\infty}^{*}$ configurations $\widetilde{G}$ as on the right of Figure 9 , where there is no edge between $v_{-1}$ and $v_{-2}$, and where the root is the first-arriving vertex to have edges to both $v_{-1}$ and $v_{-2}$; these requirements are equivalent to saying that at the arrival time of the root, $v_{-1}$ and $v_{-2}$ were in different geometric components. Reuse a now-familiar argument. At time $t$ after the arrival of the root, let $N_{t}=1+$ the number of vertices $v \neq \operatorname{root} \operatorname{such}$ that $\left(v, v_{-2}\right)$ and $\left(v, v_{-1}\right)$ are both edges of the graph process; regard the " +1 " as counting the root itself. Then $N_{t}$ is a Yule process of rate $\beta_{2}=\int_{0}^{\infty} p^{2}(x) d x$. Thus at time $t$ the number of possible unordered pairs $\left\{v_{1}, v_{2}\right\}$ which give the configuration in the figure, where we allow one of $\left\{v_{1}, v_{2}\right\}$ to be the root, equals $\left(\begin{array}{c}N_{t} \\ 2\end{array}\right)$. Because the age $T$ of the root has $\operatorname{Exp}(1)$ distribution, we see

$$
\bar{\chi}(\widetilde{G})=E\left(\begin{array}{c}
N_{T} \\
2
\end{array}\right) \times E Q
$$

where $\bar{\chi}(\widetilde{G})$ is the density of graphs as on the right of Figure 9, perhaps with extra edges, but subject to the requirement that the root is the first-arriving vertex to have edges to both $v_{-1}$ and $v_{-2}$. Using (28) and (57),

$$
E\left(\begin{array}{c}
N_{T} \\
2
\end{array}\right)=E\left(e^{2 \beta_{2} T}-e^{\beta_{2} T}\right)=\frac{1}{1-2 \beta_{2}}-\frac{1}{1-\beta_{2}}=\frac{\beta_{2}}{\left(1-2 \beta_{2}\right)\left(1-\beta_{2}\right)} .
$$

One can now write

$$
\overline{\operatorname{dens}}\left(K_{2,2}\right)=\frac{1}{2} \bar{\chi}\left(K_{2,2}\right)=\bar{\chi}\left(G^{*}\right)+\bar{\chi}(\widetilde{G})
$$

because a 4 -vertex graph in $\mathcal{G}_{\infty}^{*}$ containing $K_{2,2}$ is either of the form $G^{*}$ or is the restriction of a graph of the form $\widetilde{G}$, in which the extra root is specified by the requirement stated above (the factor $1 / 2$ reflects the fact $\iota\left(K_{2,2}\right)=2$ ). Combining the formulas above gives

$$
\frac{1}{2} \bar{\chi}\left(K_{2,2}\right)=\frac{\beta_{2}^{2} \partial}{\left(1-2 \beta_{2}\right)\left(1-\beta_{2}\right)}+\beta^{2} \partial^{2} / 2 \times \frac{\beta_{2}}{\left(1-2 \beta_{2}\right)\left(1-\beta_{2}\right)}
$$

which simplifies to (46).

\subsection{Directed percolation}

Here we record some calculations without detailed explanation. In the context of the space-time PWIT and the evolving random graph process $\mathcal{G}_{\infty}^{*}(s)$, we can seek to grow a "core" graph $\mathcal{C}(s)$ inside $\mathcal{G}_{\infty}^{*}(s)$ via a greedy rule:

a newly-arriving vertex is included in $\mathcal{C}(s)$ if it creates an edge to some vertex already in $\mathcal{C}(s)$, in which case all such edges are included in $\mathcal{C}(s)$.

If this construction works, we expect the process $(\mathcal{C}(s))$ to have a a stationary distribution $\mathcal{C}(0)$, say, where $\mathcal{C}(0) \subset \mathcal{G}_{\infty}^{*}$. Consider

$$
\begin{aligned}
q & =P(\text { root } \in \mathcal{C}(0)) \\
Y & =\text { out-degree of root in } \mathcal{C}(0), \text { given root } \in \mathcal{C}(0) .
\end{aligned}
$$


Consider the relation

$$
\widetilde{Y}=\sum_{i=1}^{\infty} \operatorname{Ber}_{i}(q) \operatorname{Bin}_{i}\left(1+Y_{i}, p\left(\xi_{i}\right)\right)
$$

where we write $\operatorname{Ber}(p)$ for a $\operatorname{Bernoulli}(p)$ r.v. (taking value 1 with probability $p$ and value 0 otherwise). Using the recursive structure of the limit random graph process, we see that $q$ and $Y$ solve the equations (for unknown $0<q<1$ and an unknown distribution $Y$ on $\{1,2,3, \ldots\}$ )

$$
Y \stackrel{d}{=} \operatorname{dist}(\tilde{Y} \mid \tilde{Y} \geq 1) ; \quad q=P(\tilde{Y} \geq 1) .
$$

Define $p_{\text {dir-perc }}(\alpha, \lambda)$ to be the solution $q$ if it exists, and to be 0 otherwise. The interpretation of this quantity in terms of the finite random graph process $\left(\mathcal{G}_{n}, n \geq 1\right)$ is that

$$
n^{-1} E \mathbf{T} \rightarrow p_{\text {dir-perc }}(\alpha, \lambda)
$$

where $\mathbf{T}$ is the maximal size of a tree in $\mathcal{G}_{n}$ directed toward some root. So in particular, for $p_{\text {perc }}(\alpha, \lambda)$ defined at (18),

$$
p_{\text {dir-perc }}(\alpha, \lambda) \leq p_{\text {perc }}(\alpha, \lambda) .
$$

Equation (59) in principle determines $p_{\text {dir-perc }}(\alpha, \lambda)$, but to get an explicit bound we reuse an underlying idea. Because $1+Y_{i} \geq 2$,

$$
\tilde{Y} \geq \sum_{i=1}^{\infty} \operatorname{Ber}_{i}(q) \operatorname{Bin}_{i}\left(2, p\left(\xi_{i}\right)\right)=Y^{*}, \text { say. }
$$

If the equation

$$
q=P\left(Y^{*} \geq 1\right)
$$

has a solution $q>0$ then one can argue $p_{\text {dir-perc }}(\alpha, \lambda) \geq q$. But (60) is an explicit equation

$$
1-q=\exp \left(-\int\left(2 p(x)-p^{2}(x)\right) q d x\right)=\exp \left(-\left(2 \beta-\beta_{2}\right) q\right) .
$$

If $2 \beta-\beta_{2}>1$ there is a solution $q>0$, establishing (19).

\subsection{Out-degree in the case $\alpha \lambda=1$}

The special property of this case is that $p(x)=e^{-\lambda x}$. On the PWIT consider

$$
Y=\sum_{v \neq \text { root }} \operatorname{Ber}\left(e^{-\lambda d(v, \text { root })}\right) \text {. }
$$

This satisfies the same recursion (in the special case) as does $D_{\text {out }}$. But there is another way to study $Y$, which we sketch briefly. Either the root of the PWIT has no children within a small distance $\delta$; or it does have a child, and the 
distances to the other descendants of the root and of this child are independent copies of the PWIT distances. Because the effect on $Y$ of increasing distances by $\delta$ is to censor each Bernoulli success with probability $\lambda \delta$, we see that $Y$ is the stationary distribution of the continuous-time Markov chain on states $\{0,1,2, \ldots\}$ with dynamics

$$
\begin{array}{rll}
y \rightarrow y-1 & : \quad \text { rate } \lambda y \\
y \rightarrow y+\hat{Y}+1 & : \quad \text { rate } 1
\end{array}
$$

where $\hat{Y}$ is an independent copy of $Y$. One can now check algebraically that

$$
P(Y=y)=\left(1-\frac{1}{\lambda}\right)\left(\frac{1}{\lambda}\right)^{y}, \quad y \geq 0
$$

solves the balance equations for this chain. That is, $1+D_{\text {out }}$ has $\operatorname{Geo}\left(1-\frac{1}{\lambda}\right)=$ $\operatorname{Geo}(1-\alpha)$ distribution, as asserted in (36).

Remark. Antar Bandyopadhyay (personal communication) has given a purely analytic verification of (36).

\subsection{Triangle density of a function of degree}

Here we outline an argument for (16). Because $D_{\text {in }}$ has power-law tail and $D_{\text {out }}$ has geometric tail, when $D=D_{\text {in }}+D_{\text {out }}$ is large, say $k$, then $D_{\text {out }}=O(1)$ and $D_{\text {in }}=k-O(1)$. It is then not hard to argue that the large- $k$ behavior for $C(k)$ will be the same as for

$$
C^{*}(k)=\frac{E\left(\text { number of triangles with in-vertex } v_{0} \mid v_{0} \text { has in-degree } k\right)}{\left(\begin{array}{c}
k \\
2
\end{array}\right)}
$$

where the in-vertex of a triangle is the vertex with two in-edges.

Recall from section 4.2 that

$N(t)=$ number of in-edges at a typical vertex $v_{0}$ at time $t$ after its arrival

is the Yule process of rate $\beta$. Write $v_{1}, v_{2}, v_{3}, \ldots$ for the successive arriving vertices which create edges to $v_{0}$, and for $i \geq 2$ write

$$
M_{i}=\text { number of edges from } v_{i} \text { to }\left\{v_{i-1}, v_{i-2}, \ldots, v_{1}\right\} \text {. }
$$

After $v_{k}$ arrives there are $M_{2}+M_{3}+\ldots+M_{k}$ triangles with in-vertex $v_{0}$. If we can show

$$
E M_{k} \rightarrow b \text { as } k \rightarrow \infty
$$

then we will have

$$
C(k) \approx \frac{k b}{\left(\begin{array}{c}
k \\
2
\end{array}\right)} \sim \frac{2 b}{k} .
$$

Here we are sliding over the fact that $1+D_{\text {in }}$ is the Yule process evaluated at an independent $\operatorname{Exp}(1)$ time $T$; conditioning this to take a value $k$ does not affect the properties used in the argument below.

Suppose vertices $v_{1}, \ldots, v_{k-1}$ have arrived and consider what edges will be created when $v_{k}$ arrives. The dynamics (22) of the space-time PWIT say 
the rate of arrival of new vertices with some one of $v_{0}, v_{1}, \ldots, v_{k-1}$ as near neighbor and at distance $\in[x, x+d x]$ from that near neighbor equals $k d x$. The index $I$ of that near neighbor $v_{I}$ is uniform on $\{0,1, \ldots, k-1\}$.

Such an arriving vertex creates an edge to $v_{0}$ with probability $p(x)$. So conditional on that event (meaning the arriving vertex is $v_{k}$ ), the distance $\hat{\xi}$ from the near neighbor $v_{I}$ and the index $I=I_{k}$ of that near neighbor satisfy

(i) $\hat{\xi}$ has probability density function $\hat{p}(x)=\frac{p(x)}{\int p(u) d u}$;

(ii) $I$ is uniform on $\{0,1, \ldots, k-1\}$.

Because $v_{k}$ will copy each of the $M_{I}$ out-edges from $v_{I}$ with probability $p(\xi)$ each, and create an edge to $V_{I}$ with the same probability, we obtain the recursion

$$
M_{k} \stackrel{d}{=} \operatorname{Bin}\left(1+M_{I}, p(\hat{\xi})\right)
$$

where $M_{1}=0$ and where we interpret the right side as 0 when $I=0$. So the limit $\lim _{k} E M_{k}=b$ solves $b=(1+b) E p(\hat{\xi})$ and so $b=\frac{E p(\hat{\xi})}{1-E p(\hat{\xi})}$. Finally,

$$
\operatorname{Ep}(\hat{\xi})=\int p(x) \hat{p}(x) d x=\beta_{2} / \beta \text {. }
$$

So $b=\frac{\beta_{2}}{\beta-\beta_{2}}$ and (16) follows from (61).

\section{Comparison with other models}

Recent complex networks models fall into two categories. In the small worlds models popularized by Strogatz and Watts, vertices are points in $d$-dimensional space, which automatically provides a metric distance between vertices, and the model uses some rule to create a random graph with short-range and longrange edges. In purely graph-theoretical models, such as the basic proportional attachment model popularized by Albert and Barabási ${ }^{7}$ the vertices have no "intrinsic structure" other than that provided by the graph; we visualize this as saying that each pair of vertices is metric distance 1 apart. In a metric copying model we visualize vertices as points in some abstract metric space, representing (in the case of web pages, say) the difference between the content of the pages, or (for people) some notion of "social distance" based on location, education, profession, interests etc of the individuals. In detail the mean-field model of distance model is used for mathematical tractability rather than any claimed realism. But the exponential growth of number of vertices with metric distance is intermediate between, and surely in many contexts more plausible than, the alternatives implicit in the two standard categories of model above.

Within graph-theoretic models, the idea of distance preferences in attachment has been explored (see [12] and citations therein). But the general idea

\footnotetext{
${ }^{7}$ but really just a minor variation of Yule's idea: see Lecture 4 of 2
} 
of combining proportional attachment with metric geometry has scarcely been explored $^{8}$, and the specific use of the mean-field model is novel.

As a technical note, the mean-field model is a zero-parameter ${ }^{9}$ model of distance. Our full network model has the two parameters $(\alpha, \lambda)$; in contrast a typical small-worlds network model has four parameters (dimension, number of short-range links, constant and exponent for probability of long-range edges).

As another technical note, the property (cf. (16) $C(k) \sim c / k$ has been proposed [17] as a criterion for identifying networks which are "hierarchical" in some sense. But in our finite- $n$ model (recall section 3.5) each vertex has qualitatively the same behavior, rather than different vertices being a priori assigned different hierarchical roles. So our model is non-hierarchical, and we are inclined to regard the criterion as ineffective $\mathrm{e}^{10}$.

The specific model studied in this paper is intended as a "general purpose" model rather than being tuned to some particular subclass of real-world networks. Having as one ingredient the now-familiar proportional attachment feature, one could look at the many existing variant models in the literature and explore them within our platform. In other words, there are many ways to add a third parameter intended to express some presumed real-world feature or some theoretical desideratum. For instance

- One can impose connectivity by requiring that a new vertex always links to its nearest neighbor.

- one can add rules allowing a new vertex to immediately acquire in-edges, or for edges to randomly appear between existing edges. Such rules can be designed (as in e.g. 7] section 11) to produce power law distributions for in-degree.

\subsection{Concluding remarks}

In this paper we have focused on

- describing the model and its conceptual background (section 3)

- listing explicit formulas (sections 2.2-2.6) and exhibiting the calculations which lead to these formulas (sections 4 and 5 ).

We are postponing to a later paper consideration of

- technical issues in the relation between the finite- $n$ model and its infinite limit $\mathcal{G}_{\infty}^{*}$

- the open problems indicated in sections 2.7 - 2.8 whose study requires the "bounding" techniques of theoretical mathematical probability rather than explicit calculations.

\footnotetext{
8 gives a simulation study of an explicitly power-law model, as well as interesting empirical study of a notion of lexical distance between web pages

${ }^{9}$ Zero dimensionless parameters, to be pedantic

${ }^{10}$ One could alternatively regard it as indicating some subtle emergent hierarchical structure; cf. 19
} 
Acknowledgement I thank an anonymous referee for detailed constructive comments.

\section{References}

[1] R. Albert and A.-L. Barabási. Statistical mechanics of complex networks. Rev. Mod. Phys., 74:47-97, 2002.

[2] David Aldous. Notes on $\quad$ STAT 206. http://www.stat.berkeley.edu/users/aldous/Networks/index.html 2003.

[3] David Aldous and Allon G. Percus. Scaling and universality in continuous length combinatorial optimization. Proc. Natl. Acad. Sci. USA, 100:11211$11215,2003$.

[4] D.J. Aldous. A stochastic complex network model. Electron. Res. Announc. Amer. Math. Soc., 9:152-161, 2003.

[5] D.J. Aldous and J. Pitman. Tree-valued Markov chains derived from Galton-Watson processes. Ann. Inst. H. Poincarè Probab. Statist., 34:637686, 1998.

[6] D.J. Aldous and J.M. Steele. The objective method: Probabilistic combinatorial optimization and local weak convergence. In H. Kesten, editor, Probability on Discrete Structures, volume 110 of Encyclopaedia of Mathematical Sciences, pages 1-72. Springer-Verlag, 2003.

[7] B. Bollobás and O. Riordan. Mathematical results on scale-free random graphs. In S. Bornholdt and H.G. Schuster, editors, Handbook of Graphs and Networks, pages 1-34. Wiley, 2002.

[8] B. Bollobás and O. Riordan. The diameter of a scale-free random graph. To appear in Combinatorica, 2004.

[9] P.G. Buckley and D. Osthus. Popularity based random graph models leading to a scale-free degree sequence. Discrete Mathematics, to appear, 2004.

[10] S.N. Dorogovtsev and J.F.F. Mendes. Evolution of networks. Adv. Phys., 51:1079-1187, 2002.

[11] S.N. Dorogovtsev, J.F.F. Mendes, and A.N. Samukhin. Structure of growing networks with preferential linking. Phys. Rev. Lett., 85:4633-4636, 2000.

[12] J. Jost and M.P. Joy. Evolving networks with distance preferences. Physical Review E, 66:036126, 2002.

[13] P.L. Krapivsky, G.J. Rodgers, and S. Redner. Degree distribution of growing networks. Phys. Rev. Lett, 86:5401-5404, 2001. 
[14] F. Menczer. Growing and navigating the small world web by local content. Proc. Natl. Acad. Sci. USA, 99:14014-14019, 2002.

[15] M.E.J. Newman. The structure and function of complex networks. SIAM Review, 45:167-256, 2003.

[16] M. Penrose. Random Geometric Graphs. Oxford Univ. Press, 2003.

[17] E. Ravasz and A.-L. Barabási. Hierarchical organization in complex networks. Physical Review E, 67:026112, 2003.

[18] S. Ross. Stochastic Processes. Wiley, 1983.

[19] H. Tangmunarunkit, R. Govindan, S. Jamin, S. Shenker, and W. Willinger. Network topology generators: Degree-based vs. structural. In SIGCOMM 02. ACM, 2002. http://topology.eecs.umich.edu/archive/sigcommo2.pdf

[20] G.U. Yule. A mathematical theory of evolution, based on the conclusions of Dr J. C. Willis. Philos. Trans. Roy. Soc. London Ser. B, 213:21-87, 1924. 